\title{
Cortical Circuits for Top-down Control of Perceptual Grouping
}

\author{
Maria Kon ${ }^{1}$ and Gregory Francis \\ Department of Psychological Sciences \\ Purdue University \\ 703 Third Street
}

West Lafayette, IN 47907-2004

United States

January 31, 2022

Running head: Top-down Control of Perceptual Grouping

\footnotetext{
${ }^{1}$ Corresponding author. E-mail address: mkon@purdue.edu.
} 


\begin{abstract}
A fundamental characteristic of human visual perception is the ability to group together disparate elements in a scene and treat them as a single unit. The mechanisms by which humans create such groupings remain unknown, but grouping seems to play an important role in a wide variety of visual phenomena, and a good understanding of these mechanisms might provide guidance for how to improve machine vision algorithms. Here, we build on a proposal that some groupings are the result of connections in cortical area V2 that join disparate elements, thereby allowing them to be selected and segmented together. In previous instantiations of this proposal, connection formation was based on the anatomy (e.g., extent) of receptive fields, which made connection formation obligatory when the stimulus conditions stimulate the corresponding receptive fields. We now propose dynamic circuits that provide greater flexibility in the formation of connections and that allow for top-down control of perceptual grouping. With computer simulations we explain how the circuits work and show how they can account for a wide variety of Gestalt principles of perceptual grouping, texture segmentation tasks, amodal illusory contours, and ratings of perceived groupings. We propose that human observers use such top-down control to implement task-dependent connection strategies that encourage particular groupings of stimulus elements in order to promote performance on various visual tasks.
\end{abstract}

Keywords: grouping; Gestalt; segmentation; strategy; emergent segmentation 


\section{Introduction}

One of the fundamental characteristics of human visual perception is that a pattern of light falling on the retina is interpreted as containing perceptual groups of elements. Despite more than a century of investigation (for reviews see Rock \& Palmer, 1990; Wagemans et al., 2012), and despite playing a central role in many perceptual experiences (Palmer \& Rock, 1994; Pomerantz \& Portillo, 2011), how the human visual system creates or defines perceptual groups is currently unclear. Here, we build on a proposal made by Grossberg and Mingolla (1985a) that visual elements are grouped together (in part) when they form neural representations of connections between those elements (also see Francis, 2009; Francis, Manassi, \& Herzog, 2017). The model in Francis et al. (2017) is based on work by Grossberg and Mingolla (1985a,b) who argued that connections between elements in a visual scene could dramatically alter the interpretation of those elements. For example, the stimulus in Figure 1 shows six white squares on a black background. The Boundary Contour System model in Grossberg and Mingolla (1985a,b) represents oriented boundaries (edges). In the simulation output shown in Figure 1, the strength of a boundary at a given pixel is indicated by the intensity of the color (black indicates no active boundary), and orientation is coded by color (green for horizontal, red for vertical, and blue for diagonal). Due to input from neurons having oriented receptive fields, the boundaries outline the edges of each square. In addition, there are horizontal connections between some squares. The top and bottom edges of the rightmost pair of squares link together to connect the boundaries of each square. Likewise, the top and bottom edges of the leftmost four squares link together. Grossberg and Mingolla (1985a) refer to these connections as "emergent segmentations" because they reflect the organization and arrangement of elements rather than direct visual stimulation 11 Here we refer to these "emergent segmentations" as "connections" to avoid confusion with other terminology in the proposed model, which also involves a distinct segmentation process. One interpretation of such connections is that connected elements are part of a common group. In this example, the connected elements may be regarded as a possible

\footnotetext{
${ }^{1}$ Within the framework of the model described in Grossberg and Mingolla (1985a,b), such emergent segmentations are often amodal, meaning that an observer would be aware of the connections but not explicitly see them as producing a modal experience (e.g., color or brightness). The reader can get a sense of this distinction by looking at the stimulus in Figure 1 and noticing how the elements naturally group but do not produce visible lines between the grouped elements. There are other situations where formed connections do generate modal perceptual experiences (see Grossberg \& Mingolla, 1985b).
} 


\section{Stimulus}

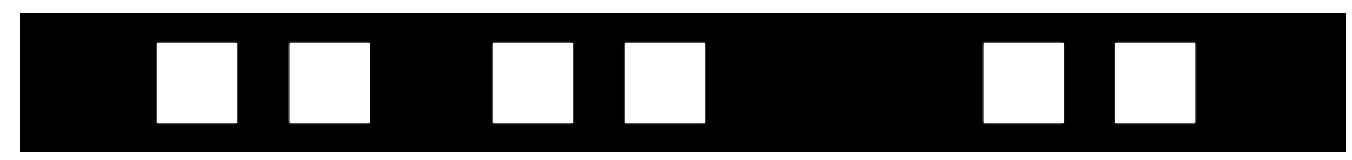

\section{Simulation}

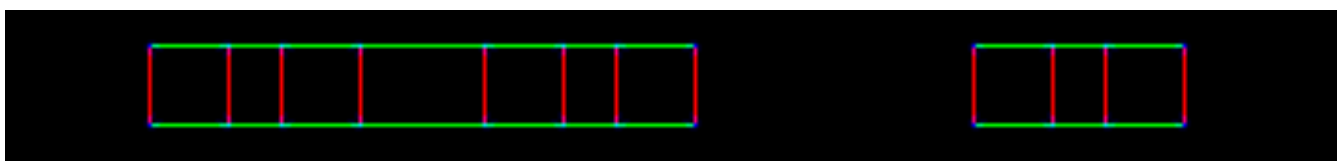

Figure 1: An example of a stimulus, which serves as input to the model, and an image that represents complex cell activity summed over 50 milliseconds of simulation time, where color represents the activity of horizontally tuned (green), vertically tuned (red), and diagonally tuned (blue) neurons at each pixel location. Black indicates that no neurons were firing at that location.

way to group the squares by proximity. Section 16 of Grossberg and Mingolla (1985a) provides several other simulation examples of perceptual grouping, and simulation examples in Francis et al. (2017) support the claim that connected elements are perceived as forming a group. This model involves a segmentation stage that separates selected elements from the rest of the scene. In Francis et al.'s (2017) simulations of various experiments, where a target vernier was flanked by distractors, connections among flanking elements allow the segmentation process to more easily select and segment these connected flankers from the rest of the scene and, thus, allow for better discrimination of the target (uncrowding).

How do connections form? Since orientation information is coded at a pixel level of an image plane, information must travel between elements. Grossberg and Mingolla (1985a,b) proposed a positive feedback circuit, where neurons with long-range receptive fields pooled information from disparate neurons that represent approximately collinear orientations and positions (e.g., two horizontally tuned neurons that are separated horizontally). These long-range neurons have a "bipole" property, so that they produce strong responses only if they receive excitatory input from both sides of their receptive fields. In turn, this bipole property allows the positive feedback circuit to interpolate connections between two disparate oriented boundaries but not to extrapolate from a single oriented boundary. This bipole behavior seems to match a variety of psychophysical (e.g., Field, Hayes, \& Hess, 1993) and neurophysiological (e.g., Peterhans et al., 1986) data. It also proves to be an important property of visual processing when explaining various aspects of depth perception (e.g., Grossberg, 1994, 2021) and dynamic perception (Francis, Grossberg, \& Mingolla, 


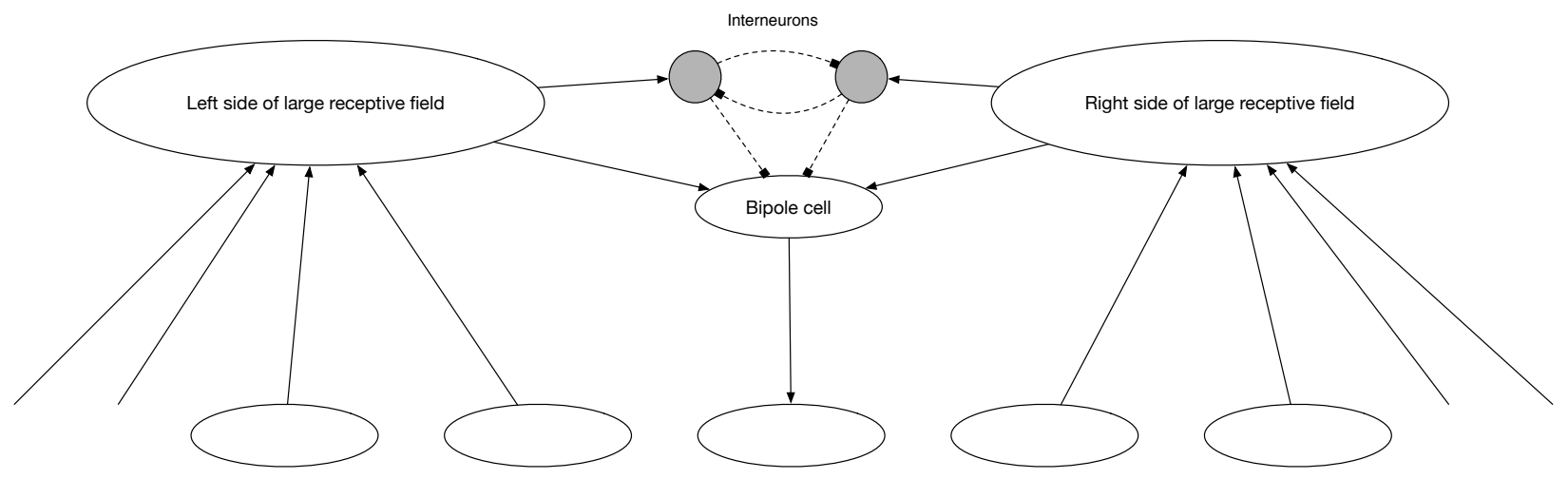

Figure 2: Schematization of a bipole circuit, which was introduced by Grossberg and Raizada (2000) and implemented in Francis et al. (2017), for a horizontally tuned bipole cell at a single pixel location. The receptive fields pool the activity of nearby horizontally tuned complex cells (bottom row) that correspond to pixel locations on either side of this bipole cell.

1994; Francis, 2009).

Early simulations of the model (Grossberg \& Mingolla, 1985a) imposed the bipole property on model simulations using nonlinear transfer functions to cap the input of each side of the receptive field and a response threshold high enough that input from both sides was required to generate output from the bipole cell. Grossberg and Raizada (2000) proposed a circuit that computed the bipole property. Figure 2 schematizes their bipole circuit for horizontally tuned neurons at one pixel location (the same circuit would be reproduced at each pixel and for different orientations). Here, the large ovals on the left and right represent neurons that receive excitatory inputs (solid lines with arrow heads) from neurons coding horizontal luminance edges (e.g., cortical complex cells in area V2). The net activity from these large receptive fields excites a common bipole cell and also excites distinct interneurons. The interneurons mutually inhibit (dashed lines with square heads) each other and also inhibit the bipole cell.

To see how the circuit produces the bipole property, first consider what happens if there is a horizontal edge feeding into the left receptive field but not into the right. The net input into the left receptive field generates excitatory input that feeds into the bipole cell but also excitatory input into its corresponding interneuron. The other interneuron is not activated because the right side receptive field does not have any input. Once the left side interneuron is activated, it inhibits the bipole cell. So, signals on just one side of the bipole cell do not produce a lasting bipole cell response. 
However, if horizontal edges feed into both the left and right sides of the receptive field, the bipole cell receives strong excitation directly from both receptive fields. Additionally, both interneurons receive excitation from their respective receptive field inputs. However, the interneurons inhibit each other, thereby reducing their total activity. Due to this mutual inhibition, the interneurons end up sending a weak net inhibitory signal to the bipole cell. Thus, with inputs on both the left and right sides of the receptive field, the bipole cell generates a strong response. Combined across many pixel locations, the bipole circuits generate completion of a connection between two closely aligned contours, but it does not extrapolate a connection from a single contour. Such behavior seems to account for many aspects of visual perception (Francis et al., 1994; Francis et al., 2017; Grossberg, 2021; Grossberg \& Raizada, 2000; Raizada \& Grossberg, 2001).

However, current versions of the bipole circuit cause connections to form only between stimulus elements that are within a particular distance from each other, e.g., they might only produce the connections shown in Figure 1. This distance is defined by the anatomy of the receptive fields. In particular, the longest "reach" of a bipole cell is defined by the extent of its receptive fields. Should a bipole cell centered between two aligned stimuli not produce a response (due to insufficient excitation to the left and right sides of the large receptive fields), then no connections will form between the stimuli. At the same time, connections form between aligned stimuli whenever they both fall within the receptive fields of a bipole cell. This behavior seems contrary to the introspective experience one has when looking at the stimulus in Figure 1. Here, it may seem natural that the shown simulated groupings reflect perceptual experience, where the four leftmost elements group together (by forming connections between them) and the two rightmost elements group together (by forming connections between them), but the left and right groups do not form connections between them. The model in Figure 2 would interpret this experience as indicating that the closest squares between the left and right groups are too far apart to engage the bipole circuit. Such limits are established by the anatomical properties of the bipole circuit in Figure 2. However, that explanation is unsatisfactory because an observer looking at Figure 1 can easily have alternative perceived groups. For example, an observer might perceive the elements as forming three pairs of squares. As yet another example, an observer might perceive all six elements as forming a single group. Finally, an observer might perceive each square as an isolated element that does not group with any other elements. These alternative perceived groupings are not possible with the anatomy of the bipole circuit in Figure 2 because it does not allow for alternative perceived groupings at all. 
These considerations motivate the need for a circuit capable of producing alternative connections. We propose that an observer may group stimuli in various ways to promote performance on a particular task. For example, if the task is to count the pairs of nearby squares, connecting the three pairs of close squares would allow for easy selection, segmentation and, thereby, identification of the target pairs. On the other hand, if the task is to identify where a natural break occurs between the stimulus elements, then it seems advantageous to form connections so that there is a left group and a right group of squares. Such connections would allow the group on one side of the image to be selected and segmented ${ }^{2}$ from the other, which enables the observer to more easily identify the location of the break.

We were inspired by the bipole circuit described in Grossberg and Raizada (2000) to develop an alternative circuit that would allow for top-down control of the spread of connections. Once we created such a circuit, we realized that it could be modified to include additional types of connection formation controls that help define groups of visual elements. The resulting control mechanisms can induce groupings that reflect many of the Gestalt grouping principles of proximity, shape, size, symmetry, and closure. In the following sections we describe the circuits and discuss how they allow for flexible perceptual groupings.

To ensure biological plausibility, we developed and simulated the model within the NEST 2.14.0 simulator program (Gewaltig \& Diesmann, 2007; Peyser et al., 2017), which provides models of various neurons and synapses and a framework to simulate networks constructed by these elements. All of our simulations used leaky integrate-and-fire neurons with alpha-function shaped synaptic currents and static synapses with manually set weights. Inputs to the network were provided by simulated direct current within the NEST program. Grossberg and Raizada (2000) previously provided links to known neurophysiology for much of the LAMINART circuit, so we do not repeat that discussion here. The new circuit is simpler than the version described in Grossberg and Raizada (2000), so many of their links should apply here as well. The proposed top-down control

\footnotetext{
${ }^{2}$ The terms "selection" and "segmentation" refer to the segmentation circuit presented in Francis et al. (2017). In that circuit, a selection signal acts as an attentional spotlight that selects all bipole cell activity it covers (and, importantly, activity that is connected to what it covers) and segments this activity, thereby isolating it from other bipole cell activity (see Francis et al., 2017, for details). Since here we focus on development of the bipole circuit, we do not further discuss the model's segmentation process, but it plays an ineliminable role in connection strategies, i.e., elements are often strategically connected to promote performance on a given task so that the target and/or distractors are easy to select and segment via the model's selection signals.
} 
circuits are novel, and we are not aware of neurophysiological investigations that reveal these precise circuits. However, there is overwhelming neurophysiological evidence (at least in mice) that topdown control influences local circuits for visual perception (Zhang et al., 2014) and modulates figure-ground perception (Kirchberger et al., 2021).

\section{A new bipole circuit}

The new bipole circuit is schematized in Figure 3. A key change is the removal of the large receptive fields. Instead, bipole cells send excitatory connections to their orientationally aligned nearest neighbors. This excitation allows for rapid spreading of connections in a direction that matches the orientation preference of the neurons (e.g., horizontally tuned cells send out horizontal connections and vertically tuned cells send out vertical connections). Without any top-down control, the interneurons in the circuit prohibit this excitatory spreading of connections but do not remove connections that have already formed between visual elements. Consider a situation where the bipole cell to the right of the labeled bipole cell in Figure 3 receives direct stimulation from a presented image. Further suppose that the bipole cell to the left of the labeled bipole cell in Figure 3 does not receive direct stimulation from the presented image. The right interneuron of the labeled bipole cell receives strong excitation from the bipole cell on the right and essentially no inhibition from the inactive bipole cell on the left of the labeled bipole cell. As a result of the net excitation for this interneuron, it sends strong inhibition to the labeled bipole cell, which prevents the spreading of activity from the cell to its right.

However, top-down control can prevent this inhibition from the interneurons, thereby allowing signals to spread. Spread is encouraged by the spread controller circuit, shown in green in Figure 3 . Top-down control of the spread controller cell consists of tuning two timing parameters: the onset of top-down input, and the duration of this input. To introduce this circuit, we initially focus on the impact of top-down control duration on connection formation. Onset is here fixed at $0 \mathrm{~ms}$, i.e., top-down control duration begins at the same time as stimulus onset. Later in this section we explain how varying the onset can impact connection formation.

When the spread controller is excited, it inhibits the interneurons and thereby encourages spread of activity among bipole cells. When the spread controller is no longer active the interneurons return to their default function, i.e., they halt the spreading of connections and, eventually, eliminate any 


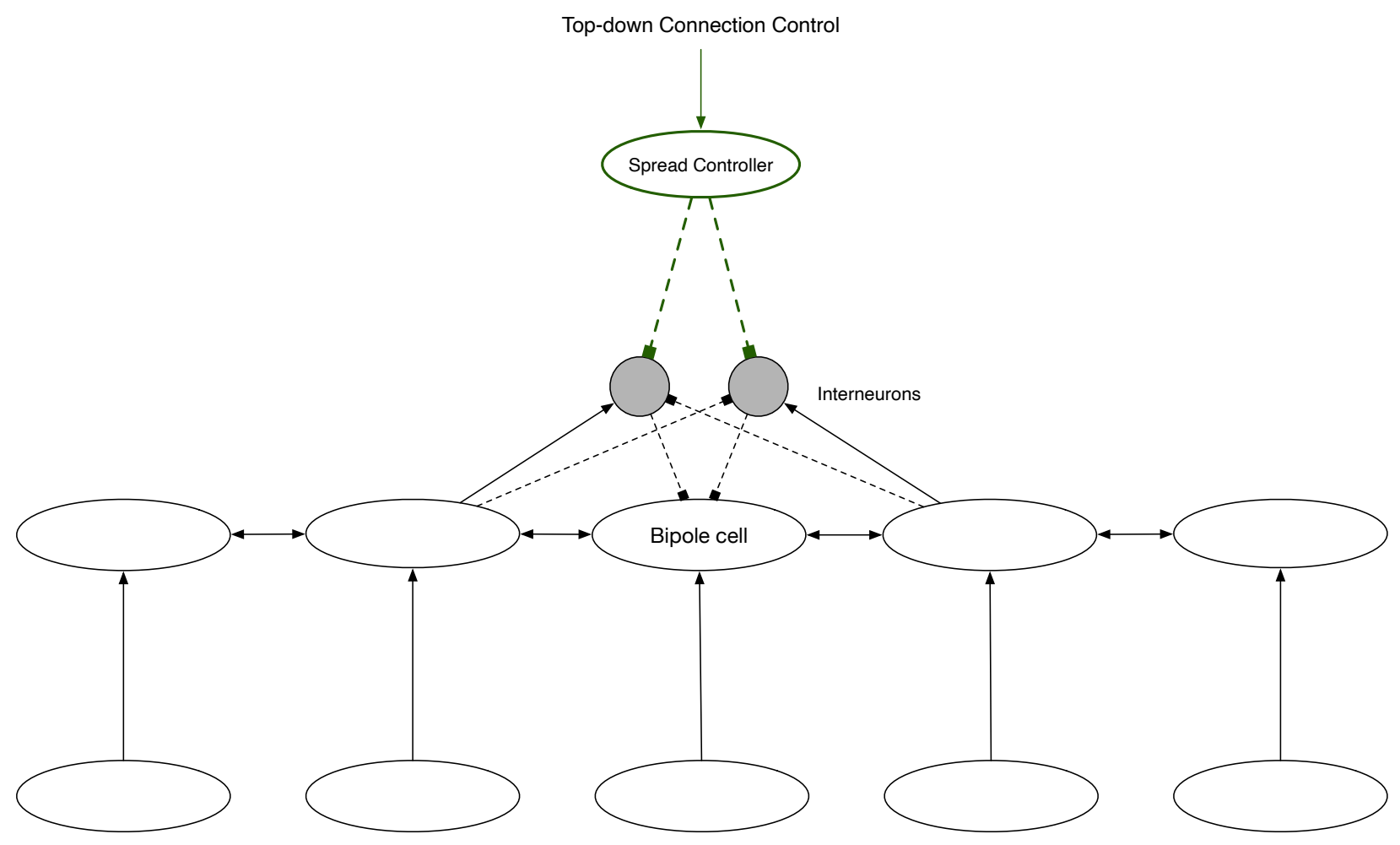

Figure 3: The modified bipole circuit with the spread controller circuit (green). The spread controller circuit allows for top-down control of the spread of connections between bipole cells via the inhibition of the interneurons for a particular period of time. The longer the interneurons are inhibited by the spread controller cell, the farther the signal from active bipole cells can spread.

created connections that have not formed a closed set.

To understand the dynamics of the circuit, consider its behavior in Figure 4. Here, the stimulus is the set of squares from Figure 1. Top-down control is imposed for a duration of 80 milliseconds (ms) beginning at the appearance of the stimulus. Because it takes time for neurons to respond to changes in stimulation (in particular for the interneurons in Figure 3 to recover from the inhibition provided by the top-down control), connections are able to spread for around $200 \mathrm{~ms}$. With these parameters, the horizontal bipole cells aligned with the top and bottom edges of the four leftmost squares connect and so do the two rightmost squares. However, not enough time has elapsed for the two groups to connect. Once the effects of top-down control have dissipated, the interneurons start to block spreading of signals. In particular, wherever there is sufficient imbalance between the inputs to the left and right interneurons of a given bipole cell, one of the interneurons will be 
activated and will inhibit its associated bipole cell. In essence, this means that the end of a line of connections erodes back to wherever there is bottom-up stimulation (Francis et al., 1994). This erosion can be seen in Figure 4 by observing how the green (horizontal) connections on the far right shrink back to the edges of the rightmost square. In contrast, connections that link stimulus driven edges remain active because each adjacent bipole cell along a connection provides nearly equivalent excitation and inhibition to each interneuron, so they hardly inhibit their bipole. For example, the connections between the middle two squares on the left remain active.

Figure 5 shows equilibrium results from five simulations, each with a different duration of top-down control excitation of the spread controller cell. For a $0 \mathrm{~ms}$ duration, there is no topdown control at all, and no connections form between the elements; the only boundaries are those generated by the stimulus edges. For a duration of $15 \mathrm{~ms}$, each square connects to its nearest neighbor. For a duration of $80 \mathrm{~ms}$, connections form among the four leftmost squares and between the two rightmost squares. For $220 \mathrm{~ms}$, all six squares connect with each other. Thus, by altering a single timing parameter, the spread controller circuit in Figure 3 allows for flexible groupings that emulate how observers can perceive Figure 1 in different ways.

Notably, these are the only connection patterns possible for this stimulus with the circuit. It is not possible, for example, to have the leftmost pair connect but the rightmost pair to not connect (because the spread control mechanism acts globally and thus is equivalent at each part of the scene). For similar reasons, it is not possible for the second from left square to connect with the third from left square without also connecting with the leftmost square. Finally, it is not possible for the leftmost square to connect with the third from left square but not with the second from left square (spreading must proceed linearly). It may be possible for people to perceive groups in these ways, but according to the model such experiences are not because of formed connections. Our proposal is that connections facilitate some perceived groups, but connections are not themselves sufficient (and, depending on the task and stimulus set, e.g., grouping by color similarity, may not even be necessary) for perceived grouping. We suggest that the experience of grouping occurs when an observer selects/segments elements, which may be connected to other elements, and notes the set of elements that are conjointly segmented with the selection (Francis et al., 2017).

So far we have seen how stimulus elements can be linked using horizontal connections. The spread controller circuit is orientation specific, i.e., it can encourage the spread of active bipole cells that are tuned to a specific orientation independently of active bipole cells with different 

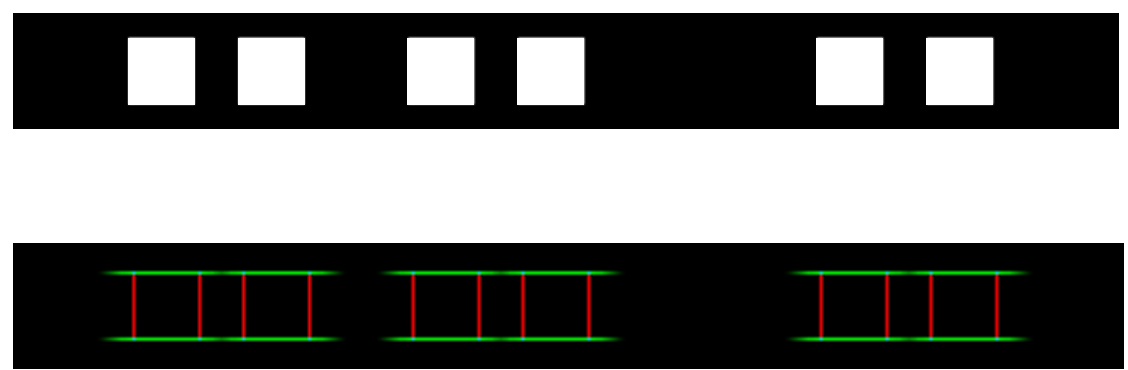

$100 \mathrm{~ms}$

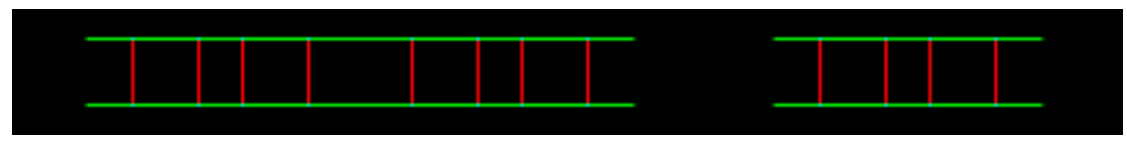

$300 \mathrm{~ms}$

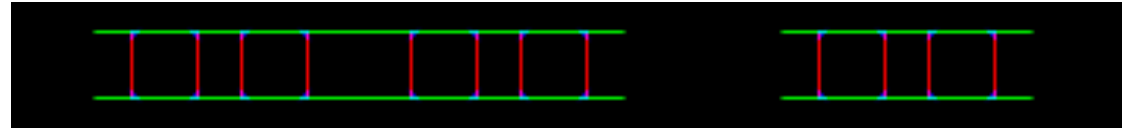

$400 \mathrm{~ms}$

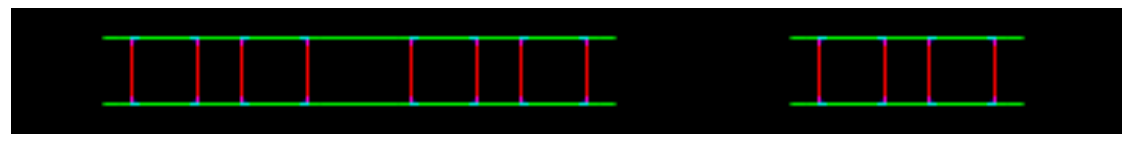

$500 \mathrm{~ms}$

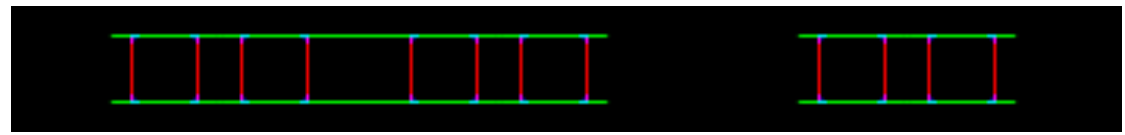

$600 \mathrm{~ms}$

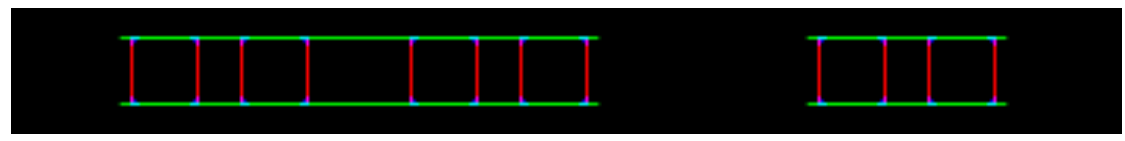

$700 \mathrm{~ms}$

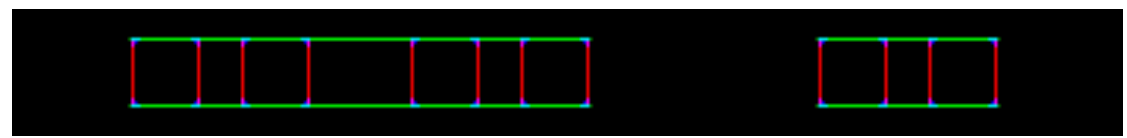

Figure 4: An illustration of the temporal dynamics of connections for a simulation where the spread controller cell is excited for a duration of $80 \mathrm{~ms}$ with onset at $0 \mathrm{~ms}$. Each image shows the activity of V2 bipole cells for a $50 \mathrm{~ms}$ interval, e.g., the first image represents activity from the time period 50-100 ms after stimulus onset. 


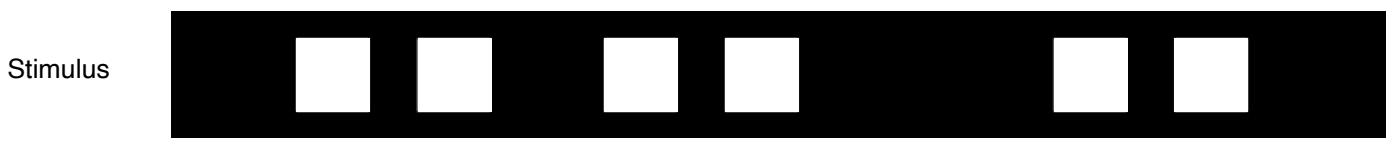

Spread Controller

Duration

$0 \mathrm{~ms}$

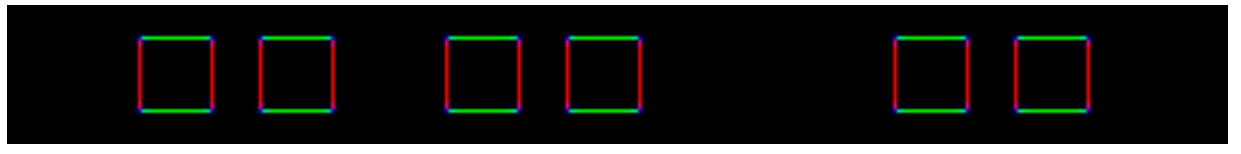

$15 \mathrm{~ms}$

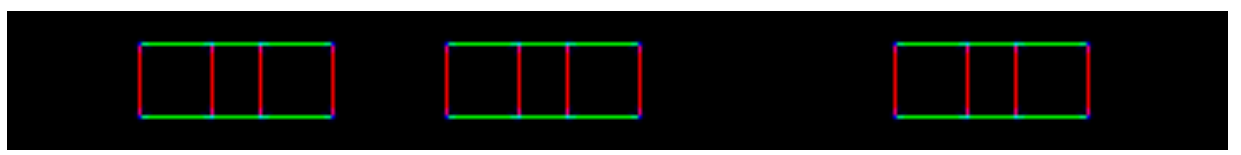

$80 \mathrm{~ms}$

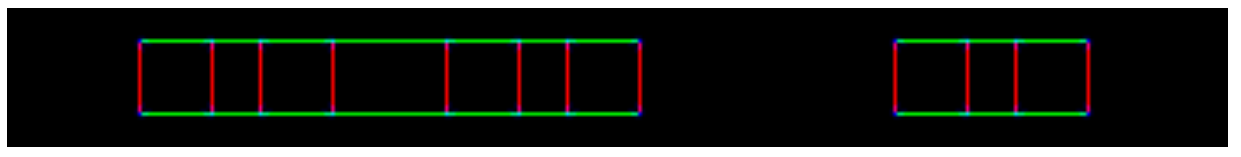

$220 \mathrm{~ms}$

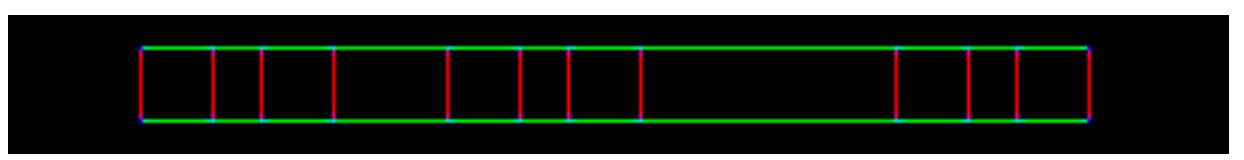

Figure 5: Altering the duration of excitation to the spread controller cell produces different connections between the detected edges for this stimulus. For each simulation, onset of top-down control excitation is at onset of the stimulus, i.e., at $0 \mathrm{~ms}$. Here each image shows bipole cell activity summed over $50 \mathrm{~ms}$ at $1700 \mathrm{~ms}$ after stimulus onset, which was chosen since it was after the simulation with the longest spread duration (220 ms) was in an equilibrium state; a duration that is high enough to connect the farthest squares causes spreading of other edges, e.g., to the left of the leftmost square, that takes longer to settle down. 
Stimulus
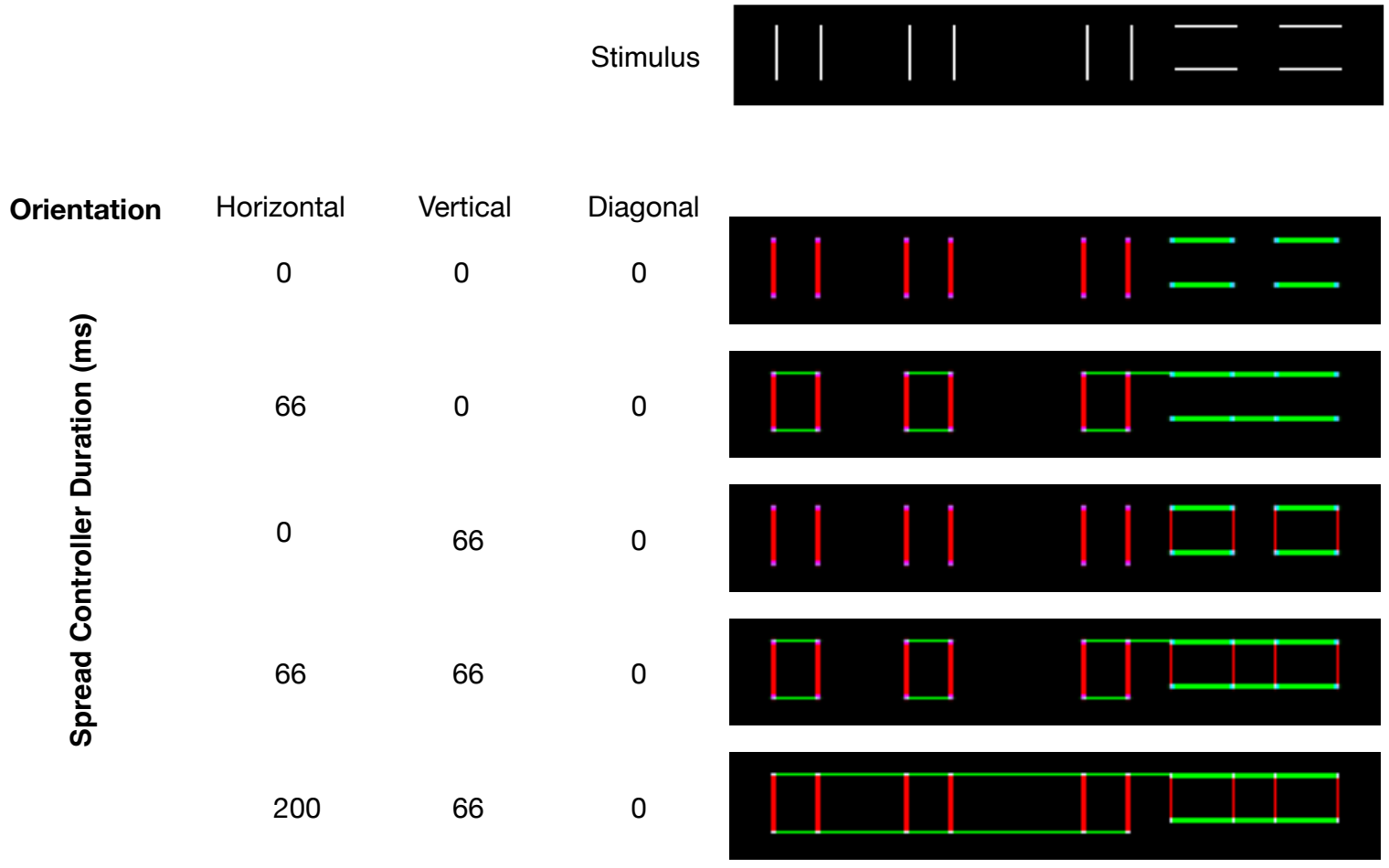

Figure 6: Demonstration that the spread of V2 connections is orientation specific and, additionally, that each orientation has its own duration of top-down control. The images show bipole cell activity summed over $50 \mathrm{~ms}$ at $700 \mathrm{~ms}$ after stimulus onset. The top-down control excitation began at stimulus onset, i.e., at $0 \mathrm{~ms}$.

preferred orientations. Figure 6 shows the connections resulting in five simulations where top-down control duration was varied for horizontal and vertical orientations. With different combinations of durations for these orientations, the circuit can promote a wide variety of connections.

For some stimuli, shifting the onset of the top-down control excitation can produce the same set of connections provided that the excitation duration is also changed. For example, Figure 7 shows that different connections can be formed among pairs of horizontal and vertical lines. The same connections can be produced with an early onset of spread control excitation (0 ms, stimulus onset) or with later onset of spread control excitation (25 ms after stimulus onset). To produce equivalent connections, the later onset requires a shorter duration of spread control excitation. This is because it takes time for activity to propagate through the system after the stimulus is shown and, in turn, bipole cell activity is relatively weak during the first $50 \mathrm{~ms}$ of stimulation. Due to this initially weak bipole signal, a longer duration of spread control input is needed to produce the 


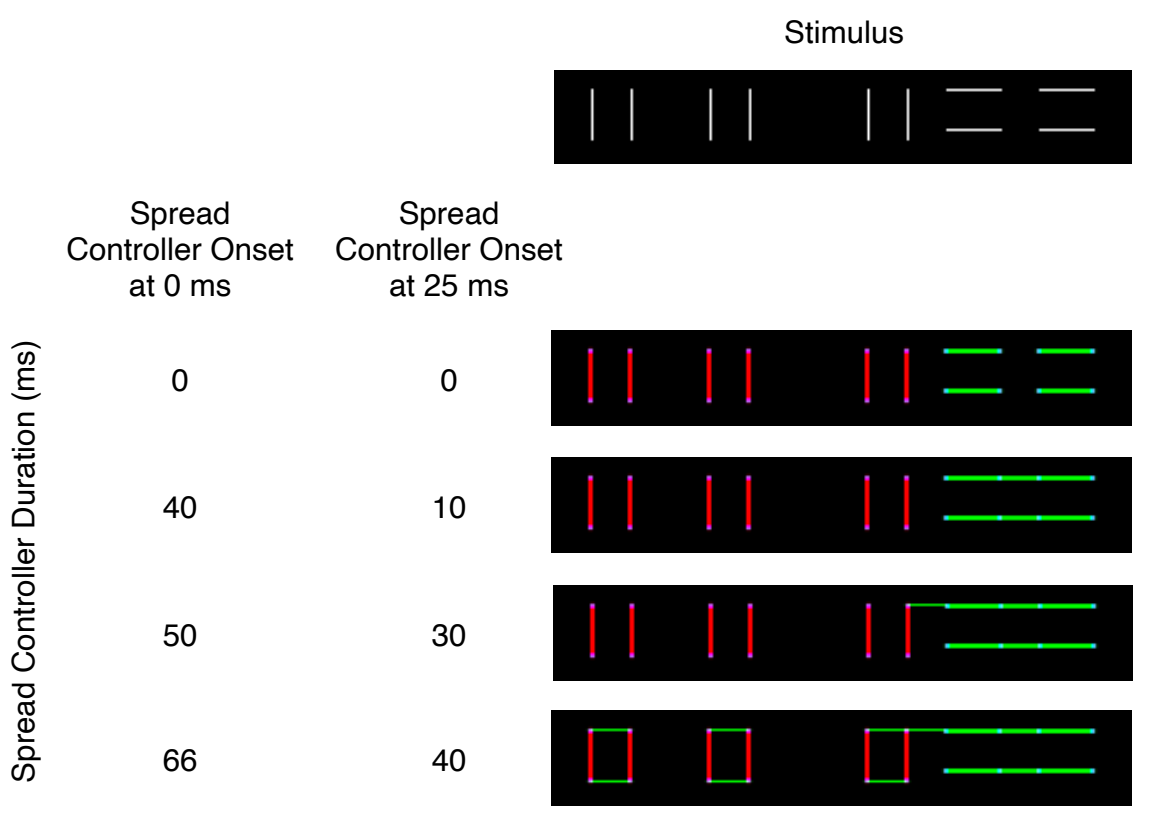

Figure 7: An example of how different combinations of onset and duration of the top-down control excitation can produce the same patterns of connections. Each image shows bipole activity summed over $50 \mathrm{~ms}$ at $700 \mathrm{~ms}$ after stimulus onset.

same connections when control input onset is at $0 \mathrm{~ms}$ compared to a simulation where input onset occurs after stimulus onset.

For other stimuli, a different onset will sometimes produce different connection possibilities. For example, consider the stimulus consisting of equidistant shapes in Figure 8. Each column shows the possible connections for a particular spread controller onset. Compare the images in each row. The connections in all but the fourth row are identical, which is similar to the results in Figure 7, where different combinations of onset and duration produce the same connections. In row 4 of Figure 8 , the left image has connections between the square and circle, while the right image does not. Moreover, the left image lacks connections between the $\mathrm{H}$ and $\mathrm{X}$, while there are such connections in the right image. In fact, there is no duration with onset at $0 \mathrm{~ms}$ that produces the connections shown in row 4 of the right column, and similarly for an onset of $25 \mathrm{~ms}$ and the connections in row 4 of the left column. This is due to the boundary signal being relatively weak at the beginning of a simulation. The single pixel horizontal edge of the $\mathrm{H}$ top/bottom is quite weak for a longer period of time than the top/bottom of the circle. Thus, a longer top-down control 


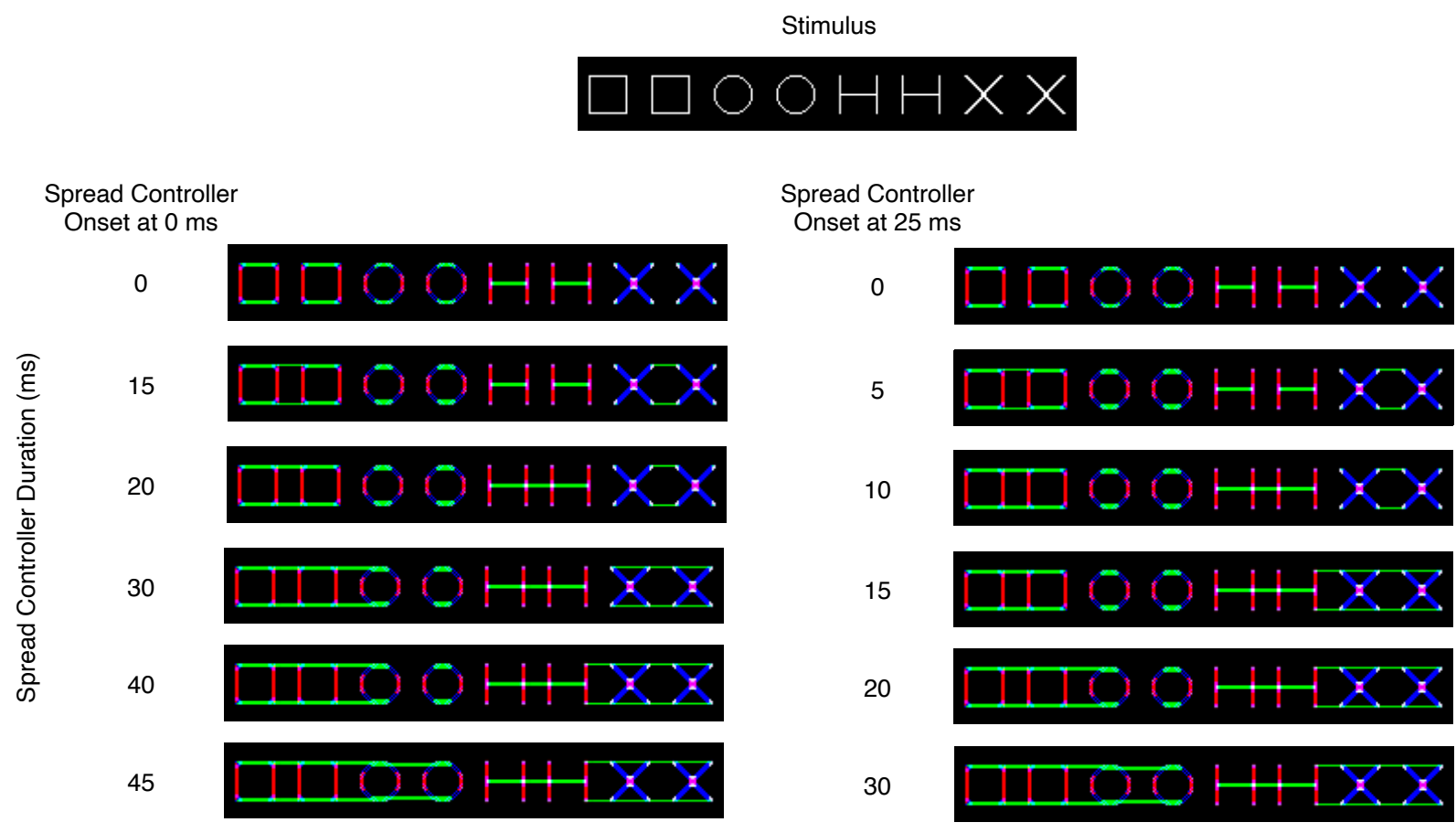

Figure 8: An example of how varying the onset of the top-down control duration can produce different patterns of connections. The images in row 4 have different connection patterns. The images show bipole cell activity summed over 700-750 ms after stimulus onset.

duration is required to connect the (weak) $\mathrm{H}$ and $\mathrm{X}$ (e.g., Figure 8, column 1, row 5), but this long duration also causes the top and bottom of the (strong) circle to connect with the squares. In contrast, with a $25 \mathrm{~ms}$ onset of the spread controller, the horizontal top/bottom of the $\mathrm{H}$ is strong enough to spread to the $\mathrm{X}$ before the top/bottom of the circle spreads to the square (because the connections from the circle must travel a longer distance). Thus, with a $0 \mathrm{~ms}$ onset, the relative strength of the circle signal allows it to overcome the handicap imposed by distance since here the $\mathrm{H}$ top/bottom is handicapped by being very weak. Similarly, differences in the activity of neurons near stimulus onset compared to later in the simulations explain why settings with the same topdown control duration but different onset do not produce identical connections. For later onsets, even with different durations, the circuits would produce nearly identical connection patterns.

Figure 9 contains parameter maps that demonstrate the abilities and limitations of the spread controller for the images shown at the top of each map. Each parameter map was created by running a simulation for each pair of values in the map. For the Figure 9 maps, the values for onsets and 
durations ranged from 0 to $60 \mathrm{~ms}$ in increments of $1 \mathrm{~ms}$. Each simulation was run for $750 \mathrm{~ms}$ because the system reached an equilibrium state by this time. We identified sets of parameter values that produced the same connection pattern and used these to produce the parameter maps. Simulations were run in parallel on three systems: a virtual machine running Ubuntu 20.04 .2 (24 cores, 60 GB RAM) on XSEDE Jetstream (Stewart et al., 2015; Towns et al., 2014), a 2021 M1 Max MacBook Pro (10 cores, 64 GB RAM), and a 2019 Intel MacBook Pro (8 cores, 32 GB RAM). Checks of the different computing systems indicate that they give the same results. All simulations were programmed using Python scripts with the package NEST 2.14.0 (Peyser et al., 2017) for creating the cells and synapses and for managing network dynamics.

Each pixel in a parameter map corresponds to a pair of onset and duration values. The color of a pixel indicates the resulting connections produced by these parameter values, and the image on/near each colored area shows the resulting bipole activity (700-750 ms after stimulus onset) and connection pattern at equilibrium. Consider the parameter map on the left. Consistent with the description of the spread controller, when spread controller duration is small, no elements connect with any other element (the black region on the bottom of the parameter map), regardless of the spread controller onset. When the spread controller duration is long (the red and green regions), every element connects with its nearest neighbor (in slightly different ways for the red and green regions, respectively). The other colored regions indicate parameter pairs that produce slightly different connections involving how the elements connect (or not) with each other.

These spread controller parameters play a similar role in the connection patterns for the stimulus image on the right of Figure 9. With a short spread controller duration, elements do not connect with each other (black region on the bottom of the parameter map). With a long spread controller duration all elements connect with their neighbors (burgundy, dark blue, purple, light purple, cyan, and magenta regions) in different ways. A discussed above, delayed onset of the spread controller signal promotes more connections even for short spread controller durations (as indicated by the negatively sloped boundary between different colored regions). The other colored regions (intermediate spread controller durations) indicate connection patterns where some elements do not connect with their neighbors.

At least for these stimuli, a given connection pattern is generally produced by a relatively large set of similar spread control parameter values. This property suggests that such connections are not "fragile" and that top-down control could reasonably be defined, even with some noise, in such 

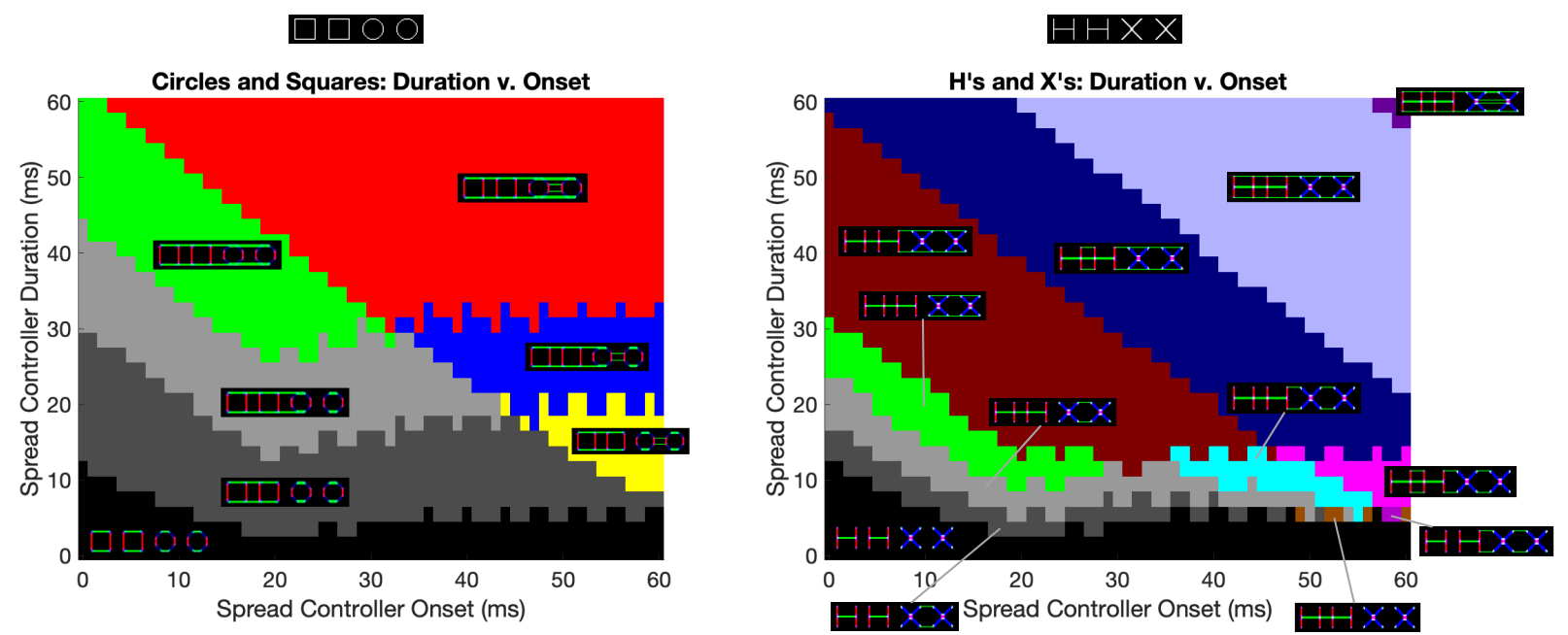

Figure 9: Parameter maps describe the possible connection patterns for a small image of squares and circles (left) and H's and X's (right). A simulation was conducted for each pair of duration and onset plotted here, where duration and onset values ranged from 0 to $60 \mathrm{~ms}$ in increments of $1 \mathrm{~ms}$.

a way as to promote a desired set of connections. However, there are a few esoteric connection patterns, such as the pattern corresponding to the brown pixels near the lower right of the H's and X's parameter map, that should be difficult to produce because they depend on a precise set of parameters.

Unsurprisingly, the spread controller seems to implement the Gestalt principle of grouping by proximity, where "close" elements are defined in various ways by the onset and duration of the spread controller. However, the variability provided by this circuit is not enough to account for how humans seem to group some sets of visual elements. In the example in Figure 7, manipulation of the spread controller cannot lead to "grouping by orientation," where there are connections only between lines of the same orientation, with no connections between any horizontal and vertical lines. The problem is that horizontal spread controller durations that allow connections to form between the tops/bottoms of the vertical lines on the left also lead to connections between the upper horizontal lines with the top of the rightmost vertical line. The stimulus in Figure 8 provides another example of how the spread controller circuit is insufficient to capture some perceived groupings, e.g., connect only each pair of same-shaped elements. This ability is oftentimes labeled an instance of the Gestalt "similarity principle." Proximity control afforded by the spread controller circuit 
does not support such an ability for this stimulus because there seems to be no top-down spread controller onset and duration that leads to connections only between same-shaped pairs.

To deal with these kinds of limitations, we speculate that there are two additional circuits: a long controller and a short controller. For particular kinds of elements, they each reduce the spread of connections induced by the spread controller and, in turn, can be used to control which elements connect. We describe both of these connection circuits below and then explain how all three circuits work together to support different kinds of connection patterns.

\section{Long and short controllers}

To provide even greater flexibility in the formation and control of connections between elements, we introduce two additional circuits that reduce the contribution to connections of bottom-up edges of specified lengths. Both of these circuits interact with the spread controller by exciting the interneurons that shut down connection formation. For the long controller, such excitation prevents boundaries of "sufficiently long" edges from spreading connections while allowing boundaries of shorter edges to spread. For the short controller, such excitation prevents boundaries of "sufficiently short" edges from spreading while allowing boundaries of longer edges to spread. In each circuit, the term "sufficiently" is established by the strength of top-down control input. Like the spread controller, the long and short controllers are both orientation specific.

Typically, the top-down input for the long or short controller is set to a fixed value for the duration of a stimulus, making the short and long controller circuits active for an entire simulation. However, the impact of these circuits depends on the timing of the spread controller, because neither the long nor short controller has much influence when the spread controller fully inhibits the interneurons (then connections spread from edges of all lengths). Thus, the long and short controllers typically influence the formation of connections by affecting the interneurons before and shortly after onset and after offset of top-down excitation to the spread controller. If spread controller onset occurs with stimulus onset, the long and short controllers typically influence connection formation only after spread controller offset, which, as is discussed in the next section, can alter what set of connections form. 


\subsection{The long controller}

The long controller prevents a long edge (consisting of many pixels) in a stimulus from generating the outward spread of connections. Figure 10 shows how the long controller integrates into the connection circuit described in Figure 3. The new components are emphasized with thick lines and orange color. A long control accumulator at each pixel for each orientation has a large receptive field that gathers inputs from many oriented cells along the direction of the preferred orientation. This accumulator is much like one of the sides of the bipole receptive field in Figure 2, except that the receptive field is now centered on the corresponding bipole cell pixel location. The size of the large receptive field only needs to be big enough to cover long enough edges. The simulations shown here accumulated inputs from a receptive field of plus/minus 25 pixels around a given bipole cell pixel. If the long control accumulator receives sufficient excitatory input, then it activates the bipole interneurons, and their activity shuts down the connection spreading process at that pixel. Without any top-down control signal, the long control circuit prevents any sufficiently strong boundaries from spreading connections.

Top-down control, i.e., long controller input, enables the spread of connections from a long edge by exciting the long controller cell, which inhibits the long control accumulator. If this inhibition is sufficiently strong, then the long control accumulator does not drive the interneurons and connections can spread from a long edge of input signals. For moderate values of long controller input, connections will spread for short edges but not for long edges. The effect of different long controller input values can be seen in Figure 11, left column. Here, the horizontal spread controller onset and duration were $25 \mathrm{~ms}$ and $40 \mathrm{~ms}$, respectively. The vertical spread controller duration was set to 0 , so no vertical connections form. For high long controller input, the length of the input edges does not impact the formation of connections (the top-down control signal completely inhibits the long control accumulator regardless of the number of signals feeding into its large receptive field). For the given spread controller duration, the very small horizontal edges at the top of each vertical line connect with their nearest neighbor. The top of the rightmost vertical line also connects with the longer horizontal lines on the far right.

With a weaker long controller input of 1.0, the spread from the long horizontal lines is reduced and can no longer reach to the top of the rightmost vertical line. This occurs because the long horizontal lines contribute a quite strong signal to the long control accumulator neurons through their long range receptive fields. These strong signals can activate the long control accumulator 


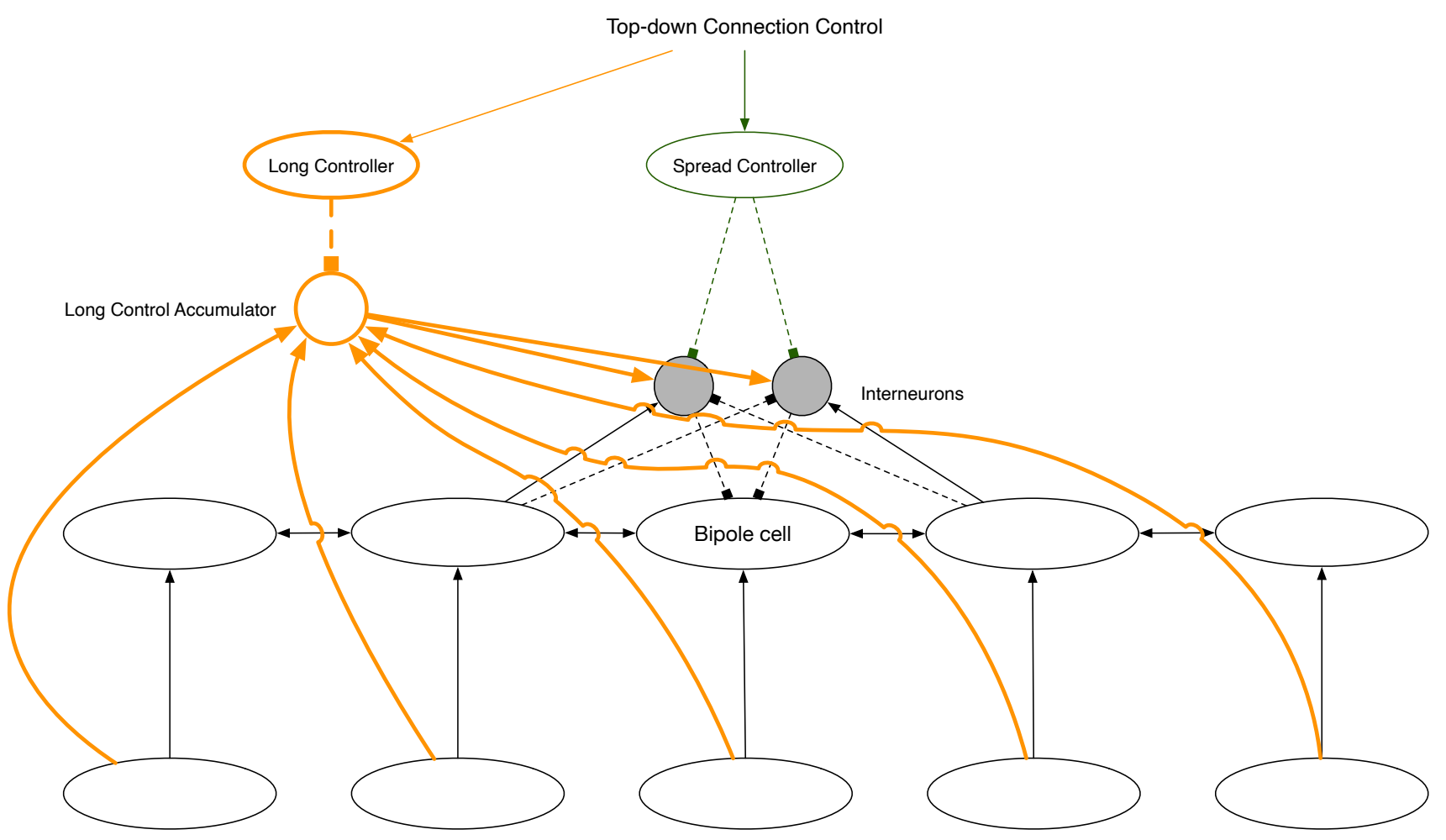

Figure 10: The components of the long control circuit for a horizontally tuned bipole cell at a particular pixel location are shown in orange. Although only two cells (bottom row) to the left and right of this bipole cell are shown here, the horizontal long control accumulator cell pools activity from 25 horizontally tuned complex cells to its left and right. 


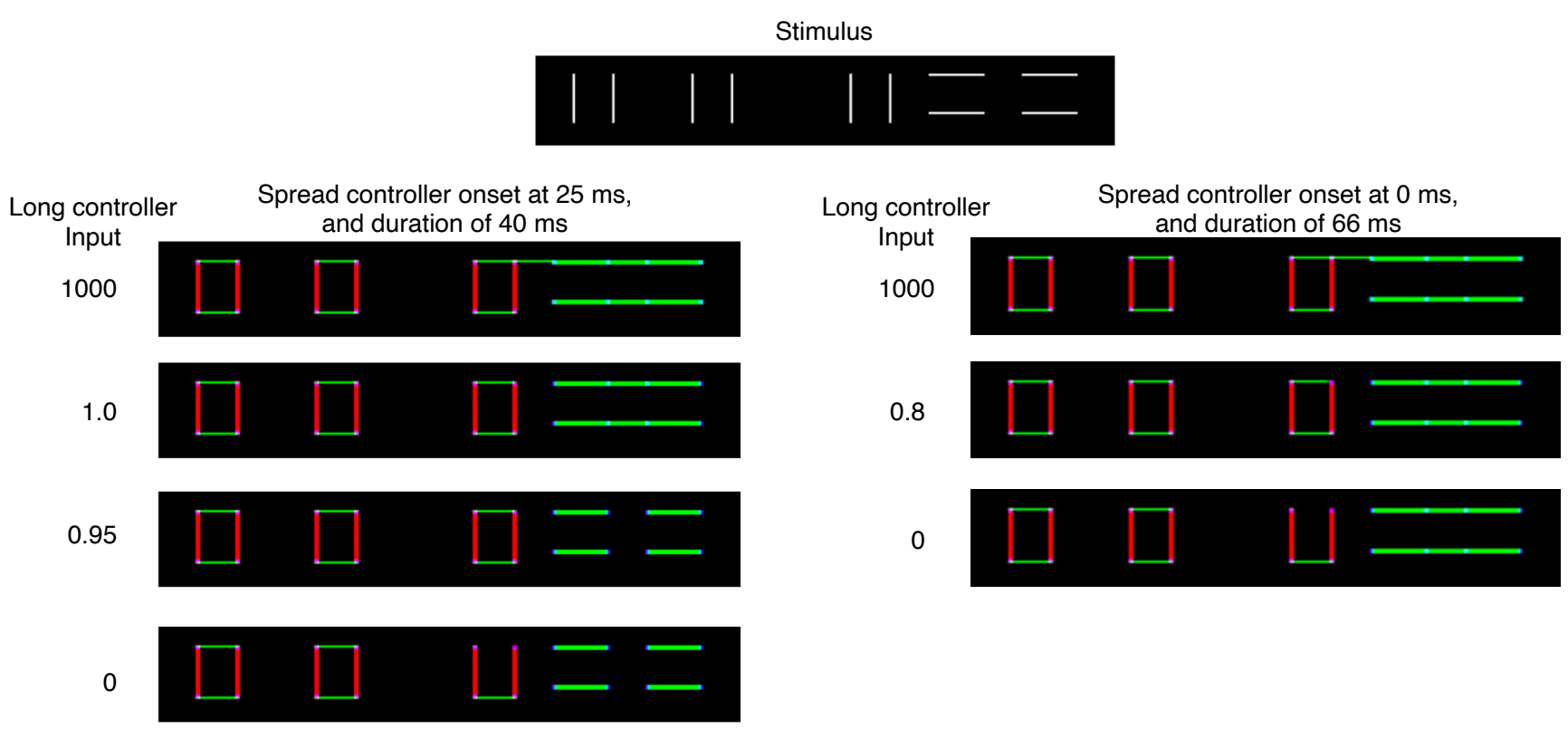

Figure 11: The connections resulting from simulations with different input to the long controller cell. The long controller circuit is effectively off when the input to the long controller is very high, e.g., 1000, and has maximum restriction when the input is 0. For each simulation in the left column, spread controller onset was at $25 \mathrm{~ms}$ after stimulus onset and had a duration of $40 \mathrm{~ms}$. For the simulations in the second column, spread controller onset was at $0 \mathrm{~ms}$ and the duration was $66 \mathrm{~ms}$.

and thereby excite the interneurons, which then shut down the formation of connections. Thus, long edges do not form connections, but short edges (such as the tops of vertical lines) do form connections. For a slightly weaker input of 0.95 , the long horizontal lines do not connect with each other. With an even weaker value of 0 , there is some "bleed" over to the top of the rightmost vertical line. The long control accumulator cells for this line register the long horizontal edge to its right. So, the small top horizontal edge does not generate connection spreading. This bleed over effect does not happen at the bottom of this vertical line because the lower horizontal lines are not horizontally aligned with the bottom of the vertical line.

In the simulations discussed thus far, the spread controller onset was fixed at $25 \mathrm{~ms}$ after stimulus onset. However, the spread controller onset interacts with the long controller to determine what connections are possible. For example, when spread controller onset is the same as stimulus onset, i.e., is at $0 \mathrm{~ms}$, a somewhat different set of connections can be produced as shown in the right 
column of Figure 11. The connections in the top two rows are essentially the same (even when having slightly different values for the long controller).

With a spread controller onset of $25 \mathrm{~ms}$, the simulations on the left side of Figure 11 show that it is possible (with long controller input between 0 and 0.95 ) to connect the vertical lines without connecting the long horizontal lines. Such a connection pattern is not possible when the spread controller has an onset of $0 \mathrm{~ms}$ and a duration of $66 \mathrm{~ms}$ (simulations on the right side of Figure 11). With the earlier appearance of spread controller inhibition, the interneurons are almost fully inhibited before signals from the long controller come in to play. With such strong inhibition, both long and short edges contribute to connection formation even when the long controller is at its maximum with an input of 0 (Figure 11, right column, last row). In contrast by delaying the spread controller onset by $25 \mathrm{~ms}$ and having 0 long controller input, long edges tend to generate excitation from the long control accumulator to the interneurons just before the spread controller cell inhibits the interneurons. Thus, for a short time, the long edges cannot contribute to the formation of connections. Should the spread controller remain active long enough, eventually even the long edges would contribute to connection formation. But here, the spread controller turns off before the interneurons corresponding to long edges would be fully inhibited, so the long edges never get a chance to contribute to connection formation.

While the specific connection patterns can vary a lot for different combinations of spread controller duration and long controller input, the parameter maps in Figure 12 demonstrate that the circuit behavior follows fairly simple rules that govern the connection patterns. Longer spread controller durations and larger long controller input values allow for more connections to form between elements (e.g., the red region in the upper right of the parameter map on the left side of Figure 12 ). With some fine tuning of the parameter pair (e.g., the yellow, olive green, and magenta regions), it is possible to form connections between squares and between circles, but not between a circle and a square. For very specific parameters values (e.g., the orange region around input 2.5 and duration $18 \mathrm{~ms}$ ), it is possible to form connections between a square and a circle but not between same-shaped elements. We suspect that such a connection pattern would be difficult to maintain due to cortical noise.

The parameter map for the H's and X's image on the right side of Figure 12 shows similar relationships between spread controller duration and long controller input. Most connection patterns can be produced by a set of similar parameters, but a few connection patterns are very fragile. 

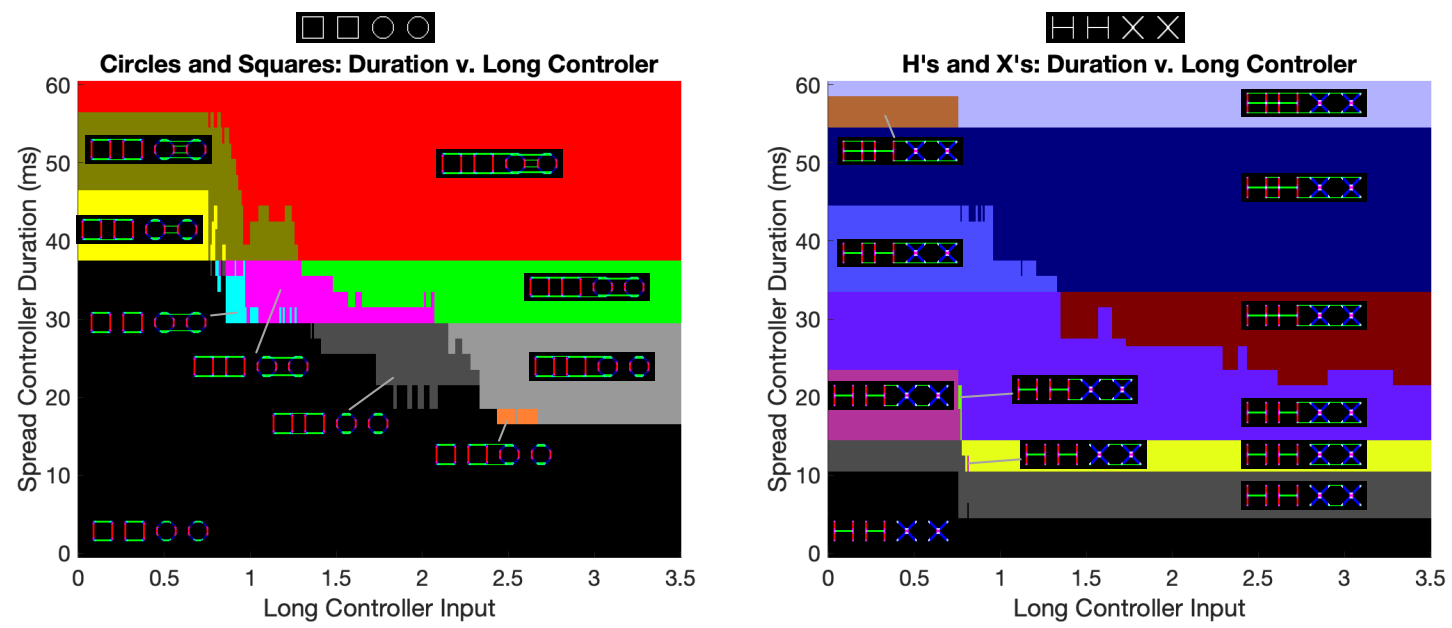

Figure 12: Parameter maps for the stimulus shown at the top of each plot. To provide a fine-grained picture of connections possible with different long controller inputs, duration ranged from 0 to 60 $\mathrm{ms}$ in increments of 1 , and long controller input ranged from 0 to 3.50 in increments of 0.01 . For all simulations here, the spread controller input was turned on $25 \mathrm{~ms}$ after stimulus onset.

Although it enables a variety of connection patterns, the long controller still has some limitations. The parameter maps indicate that the long controller may be adjusted so as to connect all and only same-shaped pairs in the stimulus with squares and circles (the yellow, olive green, and magenta areas in the left map of Figure 12, but there is no pair of parameters that form connections between the H's and connections between the X's, while not connecting the middle $\mathrm{X}$ and $\mathrm{H}$. Thus, a connection circuit with a long controller cannot connect the elements so as to facilitate grouping by shape similarity for this stimulus.

\subsection{The short controller}

The short controller circuit reduces the spread of connections from short edges. Figure 13 shows how the short control circuit integrates into the bipole circuit described in Figure 10. The new components are drawn with thick red lines. Similar to the long controller, the short controller circuit operates by exciting the interneurons of the modified bipole circuit to prevent connection spreading. This excitation is provided by the short control trigger cell, which exists at each pixel location. The trigger cell receives excitation from the complex cell at its pixel location (indirectly through an interneuron in order to equate the number of synapses for other inputs, which allows the inhibition from the short control accumulator and the excitation from the gray interneuron 


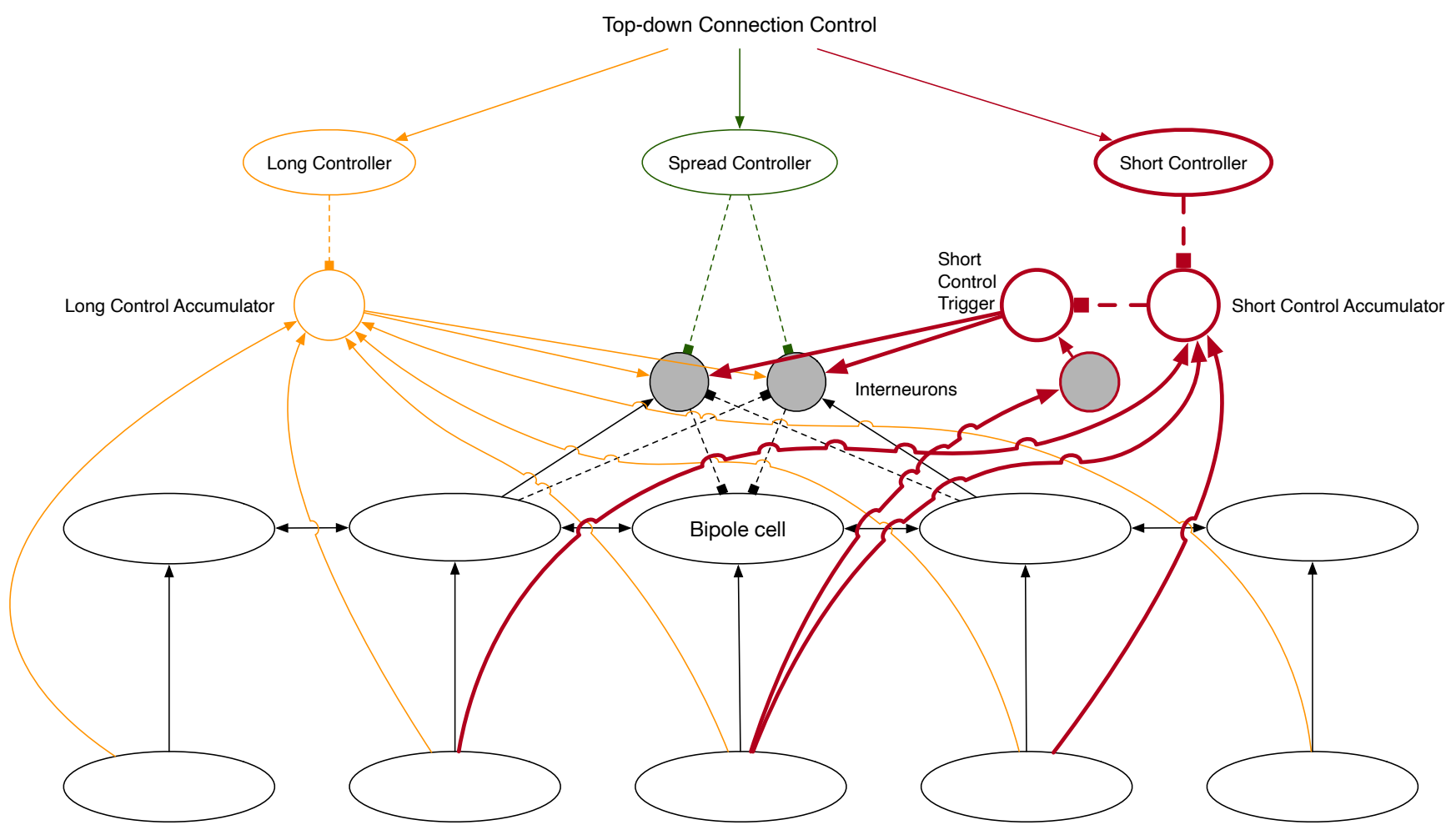

Figure 13: The components of the short controller circuit are shown in red for a horizontally tuned bipole cell. Although, for the sake of simplicity, this schematization indicates that the short control accumulator only pools the activity from two complex cells corresponding to locations neighboring that of the bipole cell (and the complex cell that feeds into the bipole cell), in the simulations each horizontal short control accumulator cell pools activity from the five horizontally tuned complex cells (bottom row) that are to the left and right of the bipole's pixel location.

with a red border to reach the short control trigger at roughly the same time). The trigger cell also receives inhibition from the short control accumulator. The accumulator gathers excitatory input from nearby pixels that align with the bipole cell's orientation preference. The size of the accumulator receptive field does not matter much; we used 5 pixels on either side of the bipole cell in our simulations.

Let us first consider the circuit's behavior with no top-down control (input equals zero). Suppose the bipole cell of interest is in the middle of a long set of edges that covers the full receptive field of the short control accumulator. The short control accumulator will be strongly excited and will strongly inhibit the short control trigger. Since the short control trigger only receives input from 
a single complex cell (the cell at the same corresponding pixel location as the bipole cell, which is directly below the cell labelled "Bipole Cell" in Figure 13), it is inhibited by the short control accumulator, which receives stronger net input. Thus, in this case, the short control trigger does not excite the bipole circuit's interneurons, so connections spread normally (depending on whether the long controller or the spread controller circuits are excited). Now, suppose the horizontally tuned bipole cell of interest is nearly isolated (say, it is the top of a thin vertical line). Then, the short control accumulator receives little excitation that is nearly equivalent to what is received by the short control trigger cell. The synapse strength between the complex cell and the short control trigger is fairly weak, so the trigger is inhibited, in net. Thus, without any top-down control, i.e., without any input to the short controller cell, the short control circuit allows spreading of both long and short edges.

The top-down short control input influences spreading by inhibiting the short control accumulator. Figure 14 demonstrates the short controller's effects when this circuit is applied to the grouping by orientation example. Horizontal spread controller onset and duration are fixed at 25 $\mathrm{ms}$ and $40 \mathrm{~ms}$, respectively. The delayed onset allows the short controller circuit to influence the interneurons as the spread controller starts to allow connections to form. As the simulations show, increasing the short controller input blocks short edges from contributing to connection formation. This control happens because the excitatory bottom-up input to the short control accumulator needs to exceed the inhibitory top-down input in order to prevent the short control trigger from halting connection spread. As shown in Figure 14, when the top-down short controller input is 1.0, the short horizontal edges at the end of each vertical line do not generate connections. This occurs because such short edges do not provide sufficient excitatory input to the short control accumulator to overcome the inhibition from the excited short controller cell, so the short control trigger halts connection spreading. In essence the magnitude of the top-down short controller input determines the definition of "short" for a given stimulus. As shown in Figure 14, with a value of 3.5, the horizontal lines on the far right are deemed "short" and do not generate connections.

Together, the long and short controllers can capture some sense of "similarity" between elements. Figure 15 shows simulation results for the row of shapes in Figure 8 . For all of these simulations, the spread controller onset is $25 \mathrm{~ms}$ with a duration of $30 \mathrm{~ms}$. As we saw before, these spread controller parameters are insufficient, by themselves, to connect all and only each same-shaped pair. The simulation results on the left side of Figure 15 vary the input to the long controller while 

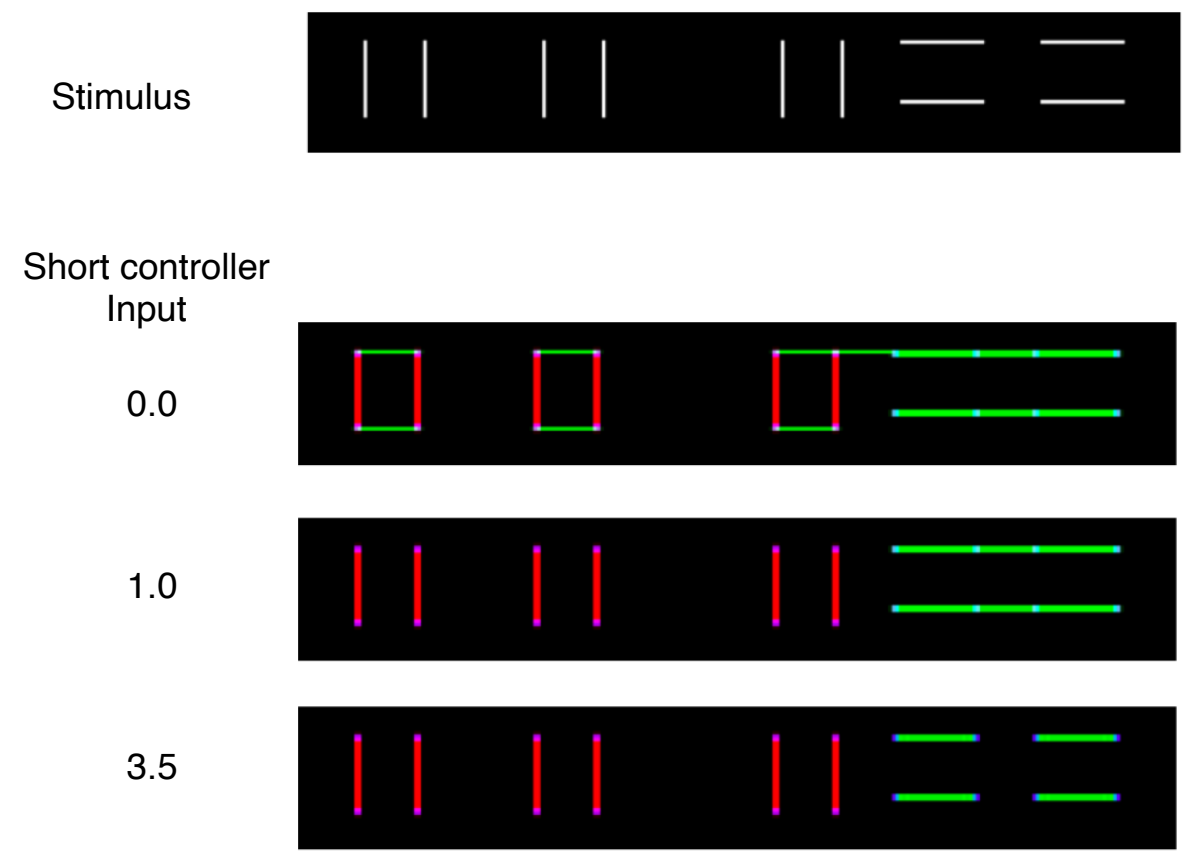

Figure 14: Connections formed for different top-down inputs to the short controller cell. Each image shows the activity of bipole cells for $50 \mathrm{~ms}$ of model time after $700 \mathrm{~ms}$ of a simulation. For each simulation, the other connection parameters were fixed: spread controller duration was set to $40 \mathrm{~ms}$ (beginning at $25 \mathrm{~ms}$ ), and the long controller circuit was effectively turned off with an input of 1000 . 


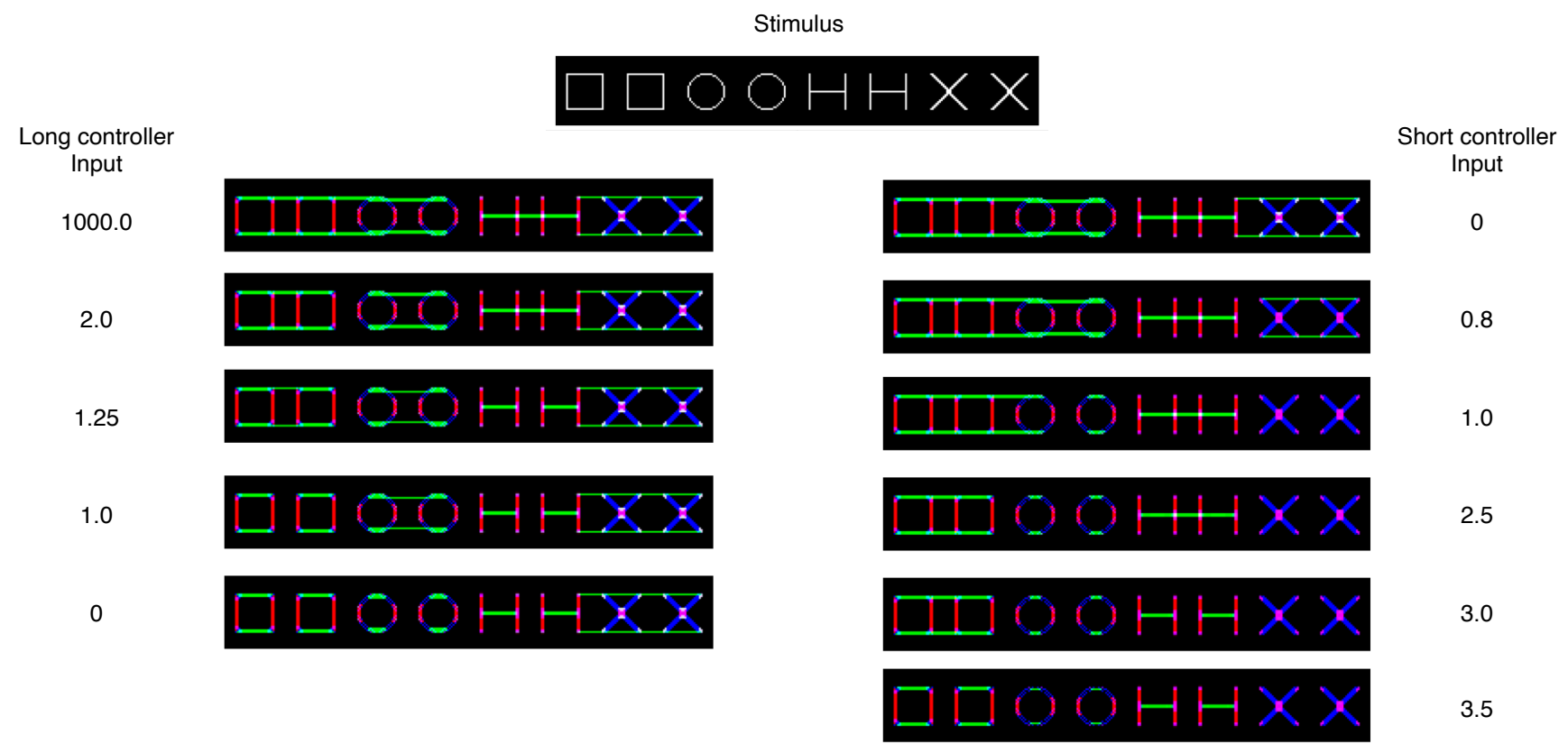

Figure 15: The impact of different inputs to the long controller (left column) and short controller (right column) for the stimulus at the top. For each simulation, spread controller duration was 30 ms and spread controller onset was at $25 \mathrm{~ms}$, i.e., spread controller parameters at which at least all similarly shaped pairs were connected. To demonstrate the effect on connections of different long controller (short controller) input values, the short controller (long controller) was turned off for each simulation in the left (right) column by having the top-down short controller input (long controller input) set at 0 (1000).

keeping the short controller input at 0 (so that the short controller does not influence connection spreading). With large values of long controller input, all edges (both short and long) can easily spread connections, so elements connect both within and between pairs. For smaller long controller inputs, long edges are less able to spread connections. This impact is most notable for the value 1.0, where the long edges of the squares no longer connect but the small horizontal edges at the tops of the X's are able to connect (but they also connect with the nearby H).

The simulation results on the right side of Figure 15 vary the input to the short controller while keeping the long controller with an input of 1000 (so that the long controller does not influence connection spreading). For small top-down inputs to the short controller, both short and long edges can spread connections, so elements connect both within and between pairs. With large values of 

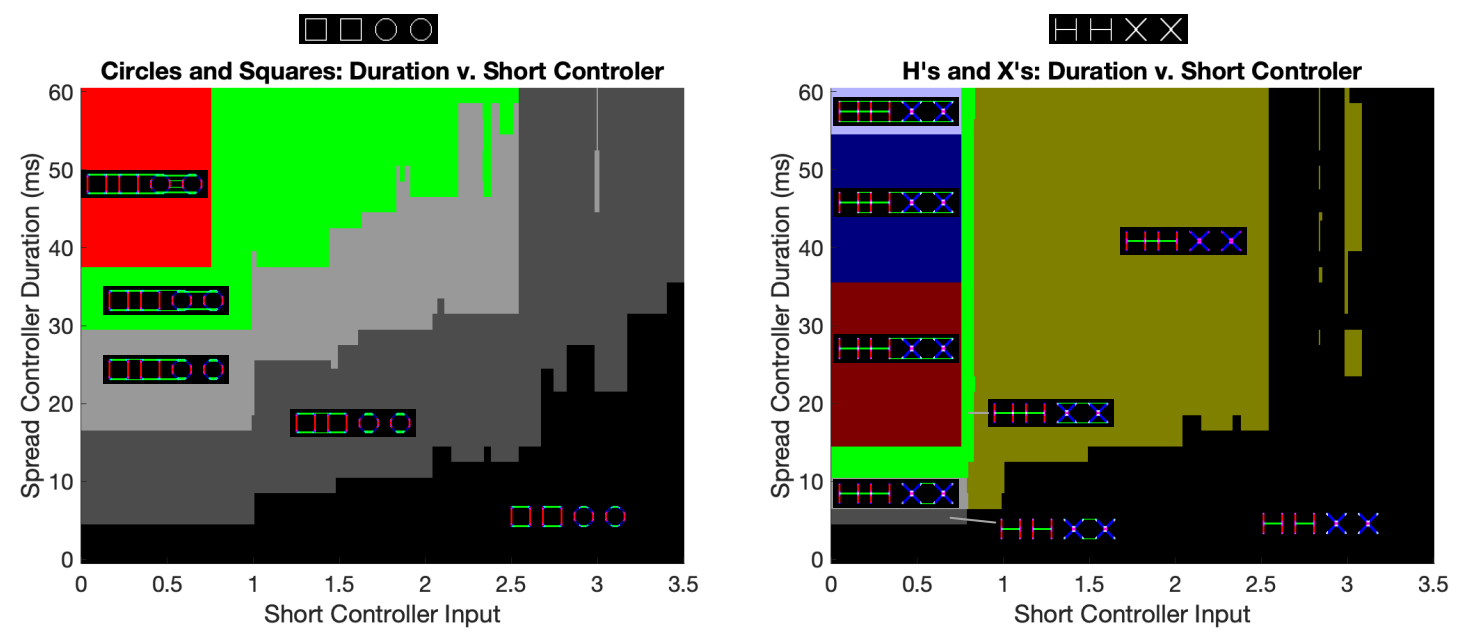

Figure 16: Parameter maps of short controller input and spread controller duration for the stimulus shown at the top of each plot. To provide a fine-grained picture of connections possible with different long controller inputs, duration ranged from 0 to $60 \mathrm{~ms}$ in increments of 1 , and long controller input ranged from 0 to 3.50 in increments of 0.01 . For all simulations, spread controller onset was $25 \mathrm{~ms}$ after stimulus onset and, thus, the second column of Figure 15 provides some examples of parameter values and resulting connections that are shown in these maps.

short controller input, only long sets of edges can spread connections. This impact is especially noticeable for the value 2.5, where the pair of squares and the pair of H's connect, but the pair of circles and the pair of X's cannot connect. For a large enough input value to the short controller (for these stimuli a value of 3.5 is sufficient), essentially all edge sets are deemed "short" and cannot spread connections.

The short controller interacts with the spread controller. Figure 16 plots two parameter maps, for different stimuli, that categorize different connection patterns for various combinations of spread controller duration and short controller input. For these simulations, the spread controller onset was always $25 \mathrm{~ms}$ and the long controller was off. The resulting connections are robust in the sense that each possible pattern of connections can be produced by many pairs of duration and short controller input values, as indicated by the relatively large areas in the Figure 16 parameter maps.

Although the short and long controllers capture some aspects of grouping by shape similarity, they are insufficient, on their own, to impose some desired groupings. In particular, if an observer wanted only the same-shaped pairs in Figure 15 to connect, they would not be able to do so only by manipulating one parameter. The next two sections show how combinations of parameters allow 
for quite complex connection patterns.

\section{Combining top-down connection controllers to promote Gestalt groupings}

By adjusting the onset and duration of the spread controller and the top-down inputs for the long and short controllers, an observer can influence what elements in a scene form connections, and thereby influence perceived groupings. For example, we already saw in Figure 11 that a combination of spread controller onset and duration and long controller input can result in connections that can be described as grouping by orientation for this stimulus. With spread controller onset at 0 ms and a duration of $66 \mathrm{~ms}$, and with long controller input of 0.8 , only the lines of the same orientation are connected together.

Figure 17 shows a situation where a combination of spread controller onset and duration and short controller input can connect stimulus elements in a way characterized by the Gestalt principle of size similarity, i.e., each adjacent same-sized pair is connected but there are no connections between the pairs. Here, the stimulus consists of a pair of large squares, a pair of small squares, a pair of large circles, and a pair of small circles. For the squares, size grouping is easy to achieve even with no short controller input because the bipole connections spread only in the direction of the orientation preference (e.g., horizontal connections only spread horizontally). Due to their difference in size, the tops/bottoms of the small squares do not line up with the tops/bottoms of the large squares, so they cannot connect. However, due to the somewhat fuzzy response of an oriented filter to the top of a circle, there is some alignment in the horizontal responses to the large and small circles. When the short controller input equals 0 , these weak horizontal signals connect across pairs. Such a grouping cannot be controlled simply by lowering the spread controller duration (here set to $55 \mathrm{~ms}$ with an onset of $0 \mathrm{~ms}$ ) because doing so would prevent the formation of connections within the same-sized circle pairs.

Setting the short controller input to a value of 0.95 makes the weak/fuzzy horizontal signal generated at the top of a circle too weak to promote connections. The input value is chosen so that just the weakest parts of the fuzzy response is blocked; the stronger responses along the circle edge are "long" relative to the top-down controlled short controller input value, and so they form connections within each circle pair. As can be seen in Figure 17, with these top-down parameters, 
Stimulus

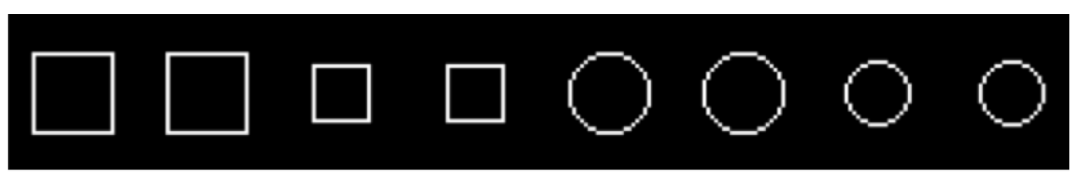

\section{Short controller Input} 0.0

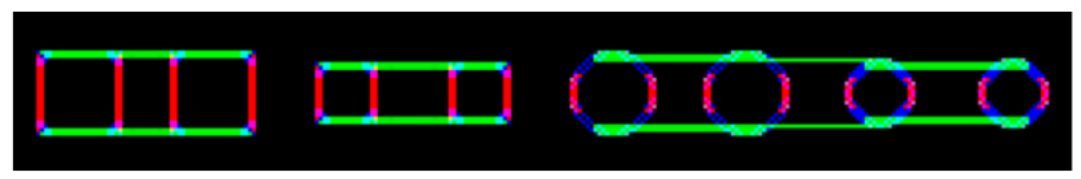
0.95

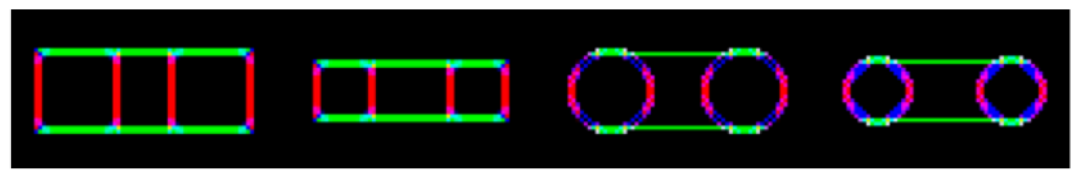

Figure 17: Model output for two simulations that have a spread controller onset of $0 \mathrm{~ms}$ and a duration of $55 \mathrm{~ms}$ for a stimulus that can be grouped by similarity of size. For the model to form connections between all and only same-sized elements, the short controller must be on with an input of 0.95 in order to prevent the connections that result between the pairs of same-sized circles shown in the middle image.

it is possible to form connections within each pair but not between pairs. It is the combination of top-down parameters that supports such groupings.

For the stimulus in Figure 8, all three connection circuits are needed to connect elements in accord with the Gestalt principle of grouping by shape similarity, i.e., all and only same-shaped adjacent pairs are connected. As can be seen in the simulation results in Figure 18, a combination of spread controller onset and duration and long and short controller inputs connects same-shaped pairs while preventing connections between pairs. Thus, with the appropriate top-down control parameters, it is possible to group the elements in this stimulus by shape similarity.

Figure 19 demonstrates that different combinations of the top-down parameters for the spread controller, long controller, and short controller circuits can lead to connections that largely emulate the Gestalt principles of grouping for a variety of stimuli. Rather than being "principles" in an absolute sense, we propose that such groupings reflect task-specific characteristics. For example, the stimulus in row 8 may be regarded as exemplifying grouping by shape similarity (Palmer 1999; 2002). We hypothesize that such a statement motivates observers to search for top-down parameters 


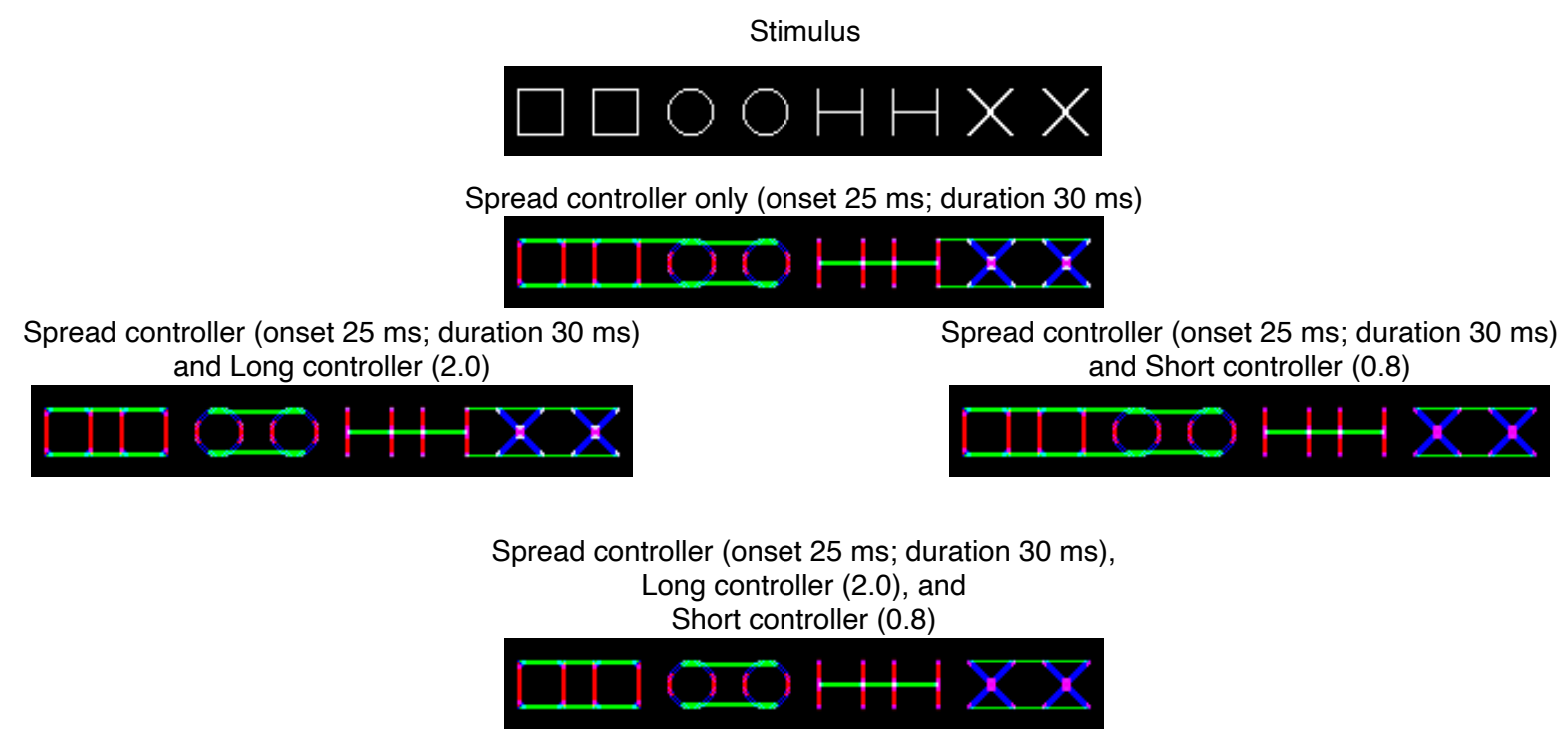

Figure 18: Model output for four simulations with different combinations of connection parameter values. For each simulation, spread controller duration is $30 \mathrm{~ms}$ with an onset of $25 \mathrm{~ms}$. The long controller input of 2.0 eliminates connections between the square and circle, while the short controller input of 0.8 eliminates connections between the $\mathrm{H}$ and $\mathrm{X}$. By combining these connection parameters, the model can produce connections that effectively group the stimulus elements by shape as shown in the bottom image. 
that form connections between shape pairs and not form connections between different shapes. The ease with which such parameters are identified may be an indication of how well the given stimulus validates, or exemplifies, the principle.

The parameter maps in Figures 9, 12, and 16 suggest that it should be fairly easy for observers to identify parameters that produce desired connection patterns. If current parameter values are insufficient to produce a desired connection pattern, the observer can change the values with a pretty good understanding of the impact. Indeed, this is precisely what we have done when producing our simulations. To generate the parameter values in Figure 19, we fixed the onset of the spread controller to match stimulus onset and then found the shortest spread controller duration where all elements of a stimulus that we wanted to connect were connected. Then, depending on whether long and/or short edges had undesired connections, we tuned the input parameter to the long and/or short controller circuit(s) to eliminate those connections. We speculate that human observers are quite skilled at tuning such parameters and do so more or less automatically.

Although sometimes used as a prescription for how elements in a scene group together, we believe the Gestalt principles are better understood as a high level description of the output of simpler underlying mechanisms. (In the Conclusions section below, we elaborate more on the relation between Gestalt grouping principles and the model.) With this view in mind, it is worthwhile to explain how the connection circuits are able to emulate the principles. In general, there are often several ways to form a desired connection pattern.

\subsection{Proximity}

The role of proximity on grouping reflects the way connections spread outward from an edge and must connect with another edge in order to maintain its representation. For the two proximity examples in Figure 19, it is easy to control the extent of spreading with the spread controller circuit so that connections form between nearby neighbors and not between farther away elements.

\subsection{Similarity of orientation}

Elements of a similar orientation tend to group together. This property reflects that the cells in a bipole circuit represent a preferred orientation. The stimulus in row 3 of Figure 19 was previously discussed at length. For the stimulus in row 4, spread controller duration for each orientation was set to $5 \mathrm{~ms}$. The short controller is on to prevent short aligned horizontal edges from connecting 


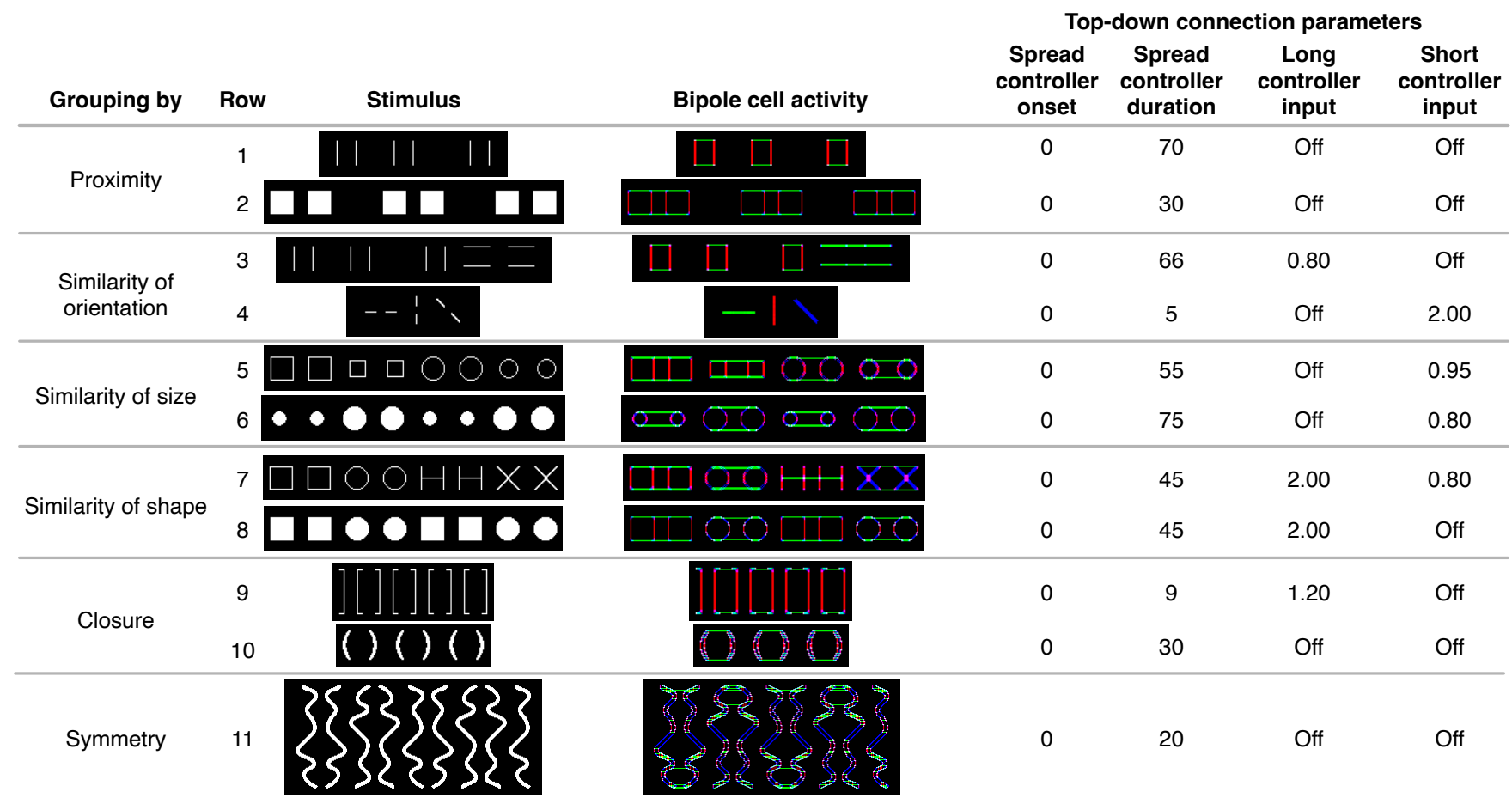

Figure 19: Grouping rules that can be accounted for by tuning the top-down control parameters of the connection circuits. The first proximity stimulus (row 1) is similar to examples in Köhler (1925). The stimuli with filled shapes (rows 2, 6, and 8) and the orientation stimuli (rows 3 and 4) are adapted from examples of "classical principles of grouping" listed by Palmer (1999; 2002). The first closure stimulus (row 9) appears in Köhler (1925), and the second (row 10) is from Pomerantz and Kubovy (1986). The symmetry stimulus (row 11) is adapted from Palmer (1999; 2002). Spread controller onset was at $0 \mathrm{~ms}$ for all stimuli. For the long controller input, "off" means that a large value (1000) is provided. For the short controller input, "off" means that a value of zero was provided. 
between the top of the upper vertical line segment and top of the diagonal line segment.

\subsection{Similarity of size}

For the stimuli in rows 5-6 of Figure 19, connections form only between elements of a similar size because only such size matches have edges that are appropriately aligned. The bipole connections cannot form between misaligned edges. With other orientations, it would be straightforward to generate other kinds of groupings for the circles in row 6 . For example, a small circle could connect with a large circle at some orientation tangent, respectively, to each circle such that they would be joined by diagonal connections. Nevertheless, it is easy to find top-down connection parameters for horizontally tuned cells that group stimuli in a way that matches the similarity of size principle.

\subsection{Similarity of shape}

As discussed above, forming connections to reflect similarity by shape requires careful setting of the top-down parameters, at least for some stimuli. Even so, for the stimuli in rows $7-8$, it is not difficult to find parameters that connect pairs of similar shapes but not different pairs.

\subsection{Closure}

In the model, elements can be grouped together if such grouping results in a "closed" set of contours. For some stimuli, such as that in row 10, this property is just a variation of the principle of proximity. Here, careful tuning of spread controller duration allows the ends of the parentheses to connect with each other in the traditional way (open on the left, closed on the right) rather than with their (slightly farther away) neighbor (e.g., closed on the left and open on the right).

Tuning of the spread controller does not fully account for the groupings in row 9 , where each bracket has the same edge-to-edge separation with its neighboring brackets. However, the presence of a vertical edge on the outside of the bracket slightly alters the response of orientation filters at the top and bottom corners of each bracket. In particular, the response of a horizontally tuned cell at the corner of a bracket is slightly weaker than the response of a horizontally tuned cell at the end of a horizontal line. This difference in strength means that a connection is more readily formed between the inside ends of brackets compared to the outside corners of brackets. This difference can be exaggerated with the long controller circuit, and this influence enables an observer to tune 
the spread controller duration and long controller input to find parameters that allow only the relatively stronger signals to connect.

\subsection{Symmetry}

Some experimental work suggests that particular symmetric elements are more likely to group together than asymmetric elements (Locher \& Wagemans, 1993; Machilsen et al., 2009). Row 11 of Figure 19 shows an example stimulus from Palmer (1999; 2002). Here people often report that mirror symmetric lines group together into pairs, with the element on the far right not being grouped with anything. Symmetry is an inherent part of the bipole circuits (as explained in Section 2 above). If the left and right sides of input to a horizontally oriented bipole cell are not of similar magnitude, the interneurons will inhibit the bipole cell and thereby not form a connection. On the other hand, the model operates on local information and so is only indirectly influenced by large-scale symmetry.

For this stimulus, the bipole cell activity in Figure 19 can connect the mirror symmetric lines into distinct pairs. These connections are formed by tuning the spread controller. The mirror symmetric lines have some parts that are closer than any non-symmetric lines. By setting the duration of the spread controller circuit, it is possible to form short horizontal connections between symmetric neighbors but not between asymmetric neighbors.

\section{Connection strategies: Tuning connection parameters to strate- gically link elements}

While the previous section indicated how tuning the connection parameters can link elements in ways that are describable in terms of classic Gestalt principles, the connection circuits can promote other groupings and, importantly, can implement connection strategies that promote performance for a particular task and stimulus set. Strategically connecting some scene elements and not others can make it easier for an observer to select, segment and, thereby, identify a target (e.g., Francis et al., 2017; Stettler \& Francis, 2018). To show how the model can implement connection strategies, we apply the model to three "demonstration" tasks. Demonstration tasks refer to instances where an image is provided in a text, e.g., the figures used by Wertheimer (1923), the table in Palmer's (1999) textbook that provides examples of different grouping principles, and the images in Beck, 
Prazdny, and Rosenfeld (1983) that demonstrate groupings of textural elements. These images are sometimes accompanied by text that functions as instructions or provides cues to the observer about what to look for in the image, e.g., the stimulus in Figure 20 is accompanied by a caption in Beck et al. (1983) that states: "short vertical lines link to form long lines, and the length of the resulting long lines makes them stand out from the smaller surrounding lines." Here we apply the model to three demonstration tasks to illustrate how connection strategies that promote performance on a given task can be implemented by the model.

\subsection{Demonstration Task 1: Beck et al. (1983)}

Beck et al. (1983) use the image shown in Figure 20 as an example of how the elements of a texture can give rise to an emergent feature. The caption of this image in Beck et al. (1983) indicates that some of its short vertical lines are linked to form long lines, and their length is an example of an emergent feature that makes them stand out from the surrounding lines. As a demonstration task, the reader looks for these long vertically connected lines. In terms of the model, the observer implements a connection strategy with the aim of generating these vertical lines. A plausible connection strategy would be to tune the connection parameters until a subset of the lines are linked via connections so that they "form long lines." By having the vertical spread controller with an onset at $0 \mathrm{~ms}$ and a duration of $20 \mathrm{~ms}$, the set of target vertical lines are connected (Figure 20. right image). Alignment and proximity are sufficient, so neither the short or long controller circuits need to be involved. This connection strategy results in a subset of the lines being connected vertically. Following Beck et al. (1983), we anticipate that these connections allow the observer to easily select and segment the target lines by supporting the segmentation mechanisms described in Francis et al. (2017).

\subsection{Demonstration Task 2: Beck (1966)}

A stimulus from a study by Beck (1966) has commonly been used as a demonstration task in subsequent work (e.g., Beck et al., 1983; Grossberg \& Mingolla, 1985a; Palmer, 1999; Rock \& Palmer, 1990). Stimulus $a$ of Figure 21 is typically presented as an example where textural segregation is stronger on the right side due to differences in orientation between the tilted T's compared to the regular T's and backward L's. The viewer is asked to examine how strongly the textures segregate or, in terms of Beck's original experimental task, where there is a more natural break in the pattern. 
Stimulus

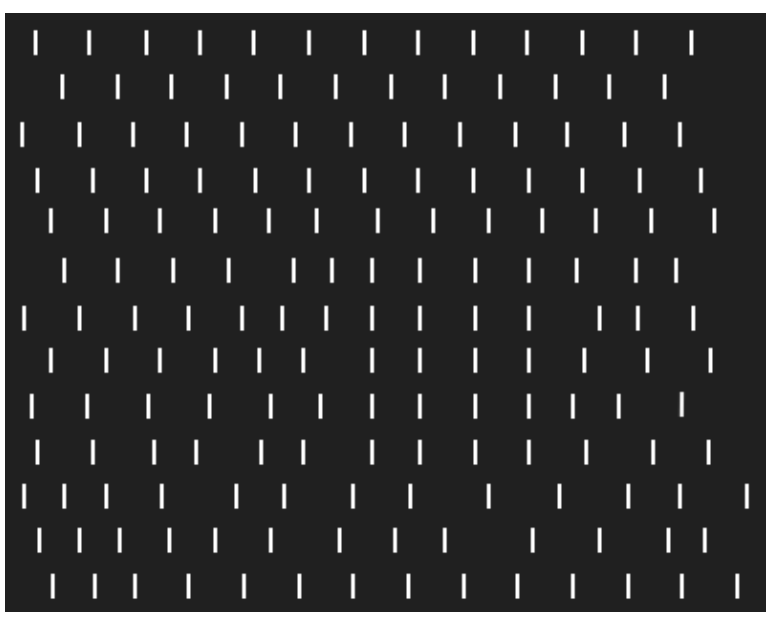

Bipole cell activity

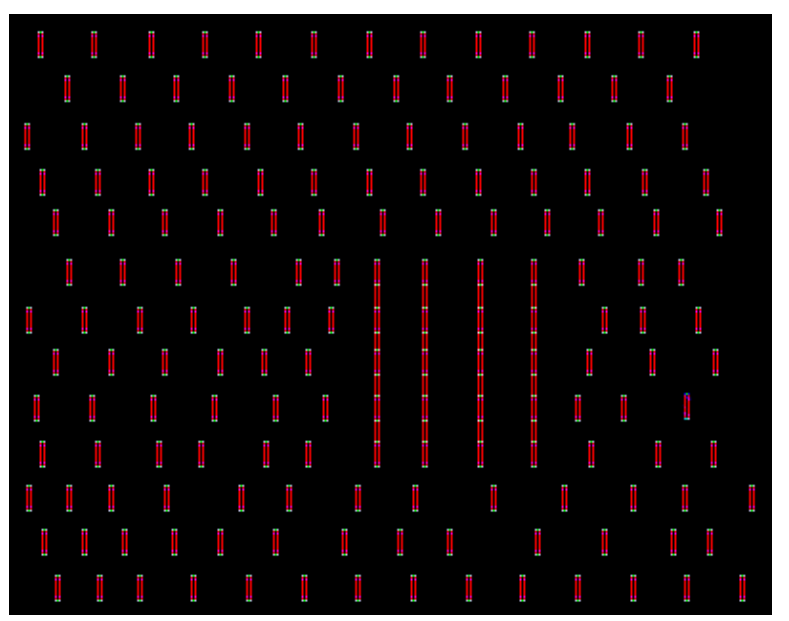

Figure 20: A demonstration task stimulus from Beck et al. (1983), and the results of a simulation that implements the connection strategy described in the text.

For this task and stimulus, a connection strategy that promotes performance would be to try to connect the elements in two of the three regions. Then, if a selection signal could cleanly segment out most of the elements of two neighboring regions, this could be regarded as indicative of there being a clean break between them and the remaining region. The top right image of Figure 21 shows a possible set of connections that results from using this strategy. For stimulus $a$, it is possible to tune connection parameters so that one set of elements does not connect with neighboring elements. Each orientation had a positive spread controller duration, and the vertical long controller and the diagonal short controller were on to prevent connections between the T's and tilted T's along their border. It was not possible to prevent the L's and T's from connecting since preventing vertical or horizontal connections between them would also prevent L's from connecting to other L's (and similarly for the T's).

Contrast this example with stimulus $e$ from Beck (1966) shown in the bottom row of Figure 21. For stimulus $e$, there is not an obvious set of connection parameters that would allow a successful connection strategy to be implemented. Since all elements consist largely of horizontal and vertical edges that are of roughly the same length, the connection parameters cannot be tuned so that elements are connected in just two of three regions. This behavior of the model corresponds with the experimental results: unlike stimulus $a$, participants did not show a significant preference for the location of a natural vertical break in stimulus $e$. According to the model, this is because an 
Stimulus
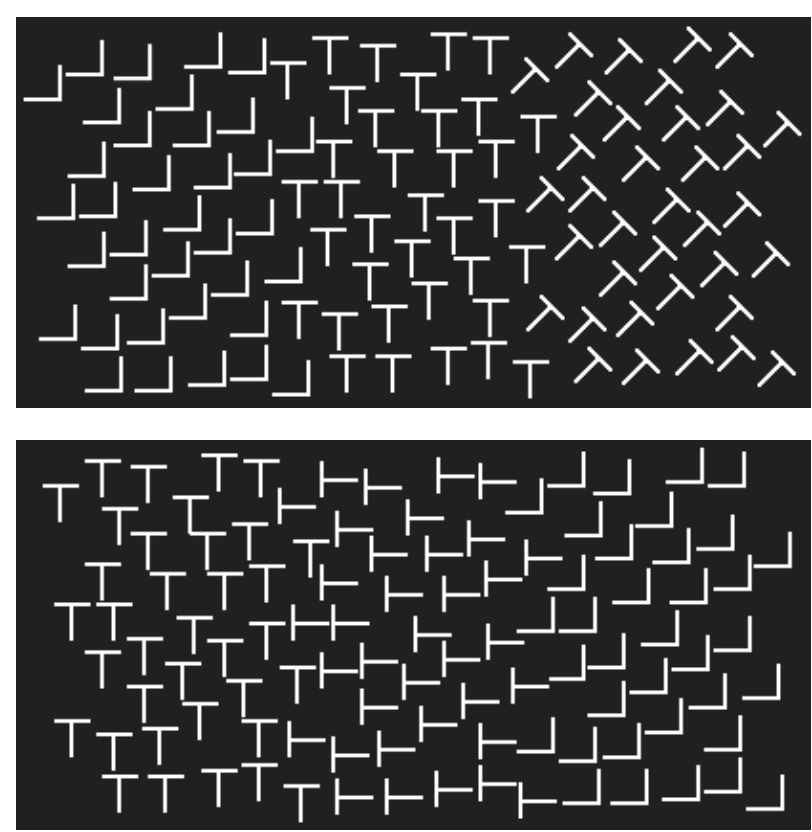

Bipole cell activity
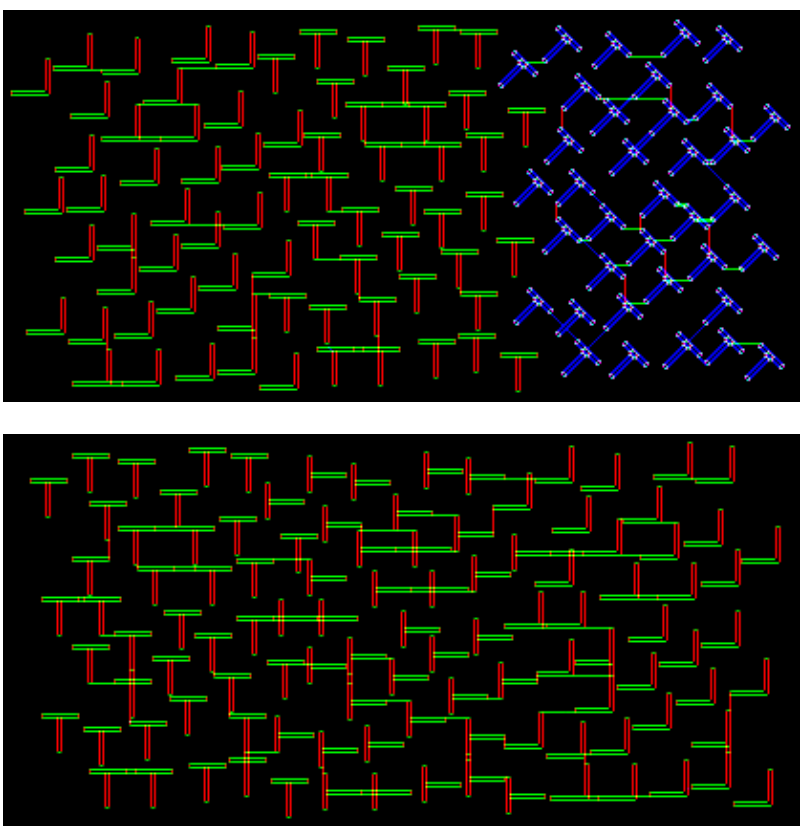

Figure 21: Stimuli $a$ and $e$ from a study by Beck (1966), and the bipole cell activity that results from implementing a connection strategy. Connection parameters were the same for each stimulus. Specifically, diagonal and horizontal spread controller durations were $30 \mathrm{~ms}$, and vertical spread controller duration was $25 \mathrm{~ms}$. Spread controller onset was at $0 \mathrm{~ms}$. The only long controller on was the vertical long controller with an input of 0.8 . And, the only short controller circuit that was on was for diagonals with an input of 1.0. 
Stimulus

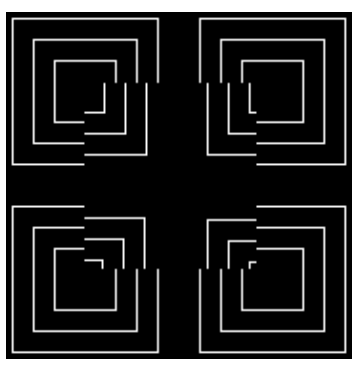

Bipole cell activity

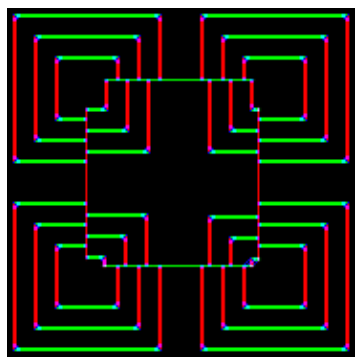

Figure 22: A stimulus used by Francis and Wede (2010) to investigate properties of offset illusory contours and the bipole cell activity that results from implementing a connection strategy that allows only short edges to form connections. The horizontal and vertical spread controller onsets were at $50 \mathrm{~ms}$ after stimulus onset and their durations were $45 \mathrm{~ms}$. The horizontal and vertical long controller parameters were set to a value of 1.0, so that only the short edges (line ends) of the offset elements were allowed to form connections. The short controller was turned off (parameter value set to zero).

observer can tune the connection parameters to implement the task-specific connection strategy of connecting elements in two of three regions for stimulus $a$ but cannot do so for stimulus $e$.

\subsection{Demonstration Task 3: Illusory contours}

Grossberg and Mingolla (1985a,b) originally developed the bipole circuit to, in part, account for the existence of illusory contours that form between inducing elements. In their model, the percept of illusory contours (e.g., Kanizsa, 1974) is a special case of emergent segmentations that are formed by connections between inducers. Such connections can trigger additional illusory experiences of brightness, color, and depth (Grossberg, 1994, 2021; Grossberg \& Mingolla, 1985a,b). Figure 22 shows that the new circuit is able to produce the connections that underly these perceptual experiences. The stimulus is adapted from Francis and Wede (2010) and uses the offset elements illusion as inducers that then generate long-range connections. The model simulation uses top-down control parameters that allow short (but not long) edges to spread a sufficient distance to form connections between line ends. As the simulation results in Figure 22 show, the resulting connections emulate the perceived illusory contour. 


\section{Application of the model to a subjective rating experiment}

While many aspects of perceptual grouping are characterized by demonstrations, quantitative investigations often ask observers to rate how well elements seem to group (or sometimes how well a group seems to "stand out" in a scene). For example, to complement their response time experiments, Palmer and Beck (2007, Experiment 2) asked observers to indicate how easy or difficult it was to see that a row of squares and circles contained a pair of repeated shapes using a 9-point scale $(1=$ very weak; $9=$ very strong $)$.

Each stimulus consisted of a row of nine shapes. Along the row the circles and squares alternated except for one repeated pair, which was the target and was either a pair of adjacent circles or a pair of adjacent squares. An element of the target pair could appear in any position in the row except for the outer two positions. There were nine spacing conditions, which are shown in Figure 23a for a square target pair. The spacing conditions were specifically designed to investigate effects of proximity by varying target pair separation (the distance between target pair elements) and nontarget pair separation (the distance between a target element and the neighboring nontarget shape). Given the influence of proximity on perceptual grouping, we would expect high ratings when the target pair separation is smaller than the nontarget pair separation (e.g., the three images in the upper right part of Figure 23a). Palmer and Beck (2007) refer to these stimuli with relatively close

target pairs as "Within-group." Likewise, we would expect low ratings when the nontarget pair separation is smaller than the target pair separation (e.g., the three images in the lower left part of Figure 23 a). These stimuli with relatively far target pairs are referred to as "Between-group." The stimuli along the diagonal of Figure 23a are referred to as being "Neutral" because each shape is equidistant from its neighbors. The left plot in Figure 23b shows the mean ratings from Palmer and Beck (2007) for the different stimuli. As expected, ratings generally increased with increasing nontarget pair separation and decreased with increasing target pair separation.

We hypothesize that judgments about how well the target elements group, relative to distractor elements, is (in part) determined in the model by the relative ease of forming connections between the target elements only. Such connections would promote selection and identification of the target items (Francis et al., 2017). If this strategic connection pattern can only be formed with a small set of connection parameters, then the rating would be low. If such connections can be formed by a large set of connection parameters, then the rating would be high. Additionally, we hypothesize 
(a)

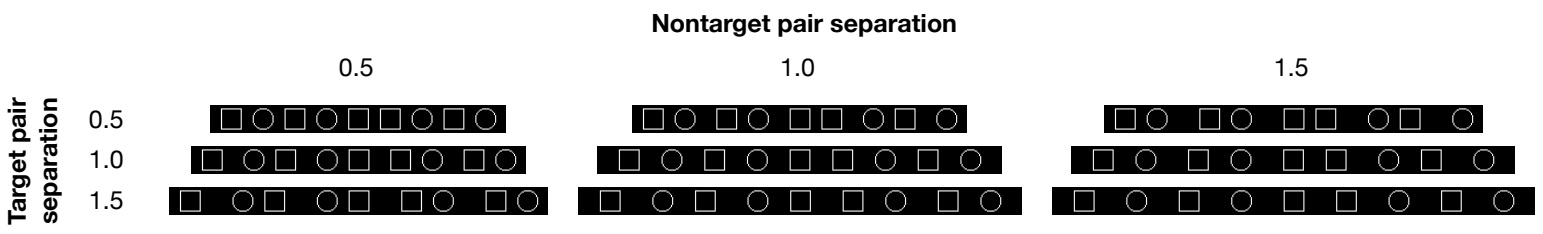

(b)
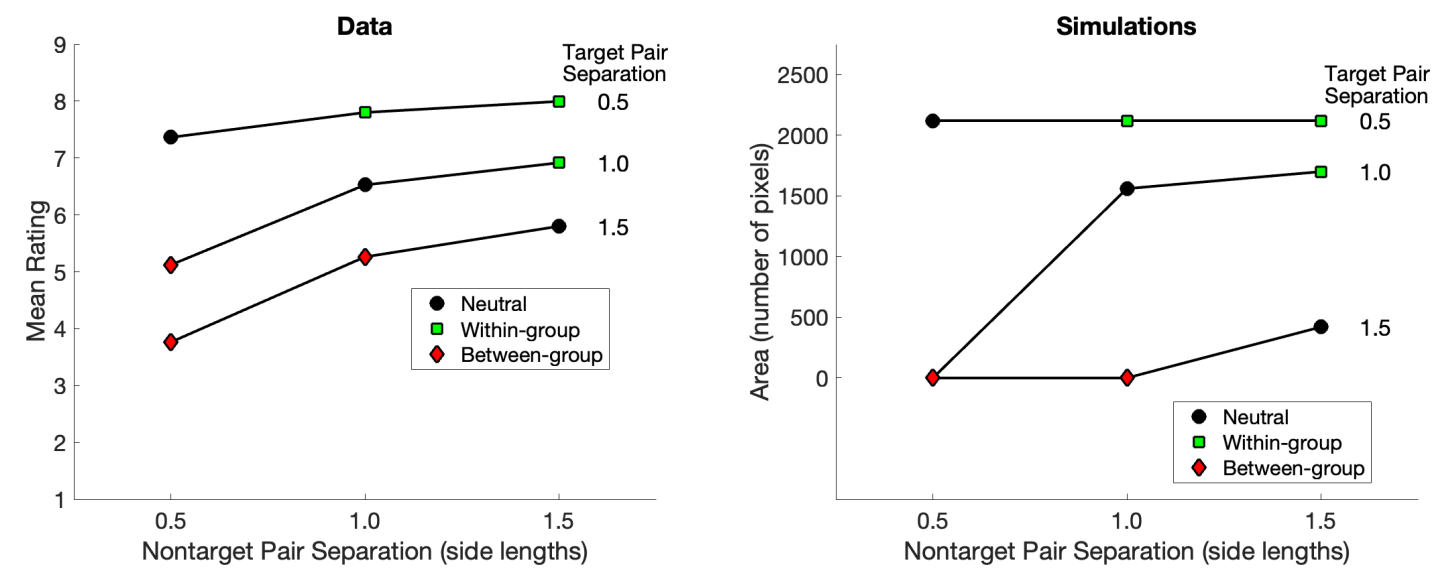

Figure 23: (a) Stimuli used by Palmer and Beck (2007) that varied spacing between neighboring elements. The target was a pair of adjacent elements with the same shape. Shown here are examples with a square target pair in positions 5 and 6, counting from the left. (b) Experimental ratings (left) and model simulations (right) for the various stimuli. Ratings reported by Palmer and Beck are high for within-group stimuli and lower for between-group stimuli, and a similar pattern is produced by the model. 
that participants made their ratings based on connection parameters that would form connections between both square pairs and circle pairs. Due to the design of the experiment (with hundreds of stimuli being presented for rating and with target shape conditions being randomly interleaved), we reason that an observer would want to make judgements relatively quickly to efficiently complete the experiment and, thus, would tune their connection parameters so they would connect the target pair of shapes (if possible) for both square and circle shapes. So, for each condition, we measured the area of the parameter map that produces the strategic connection pattern for both square and circle shapes. If this area is large, then it is indicative of a high rating. If this area is small, then it is indicative of a low rating.

Through other simulations, we discovered that the short controller plays no role in establishing the strategic connection pattern for these stimuli, so we turned it off for all simulations. We also set the onset of the spread controller to $0 \mathrm{~ms}$, so that it started with the onset of the stimulus. With these constraints, we then ran repeated simulations that varied the duration of the spread controller and the input of the long controller. These parameter maps were generated in the same way as for the parameter maps presented above, except that the simulation duration was longer (1000 ms) to ensure equilibrium.

We created one parameter map for each spacing condition and target pair shape. Although Palmer and Beck (2007) also varied the location of the target pair, we only used stimuli with the target pair in the positions shown in Figure 23a. Palmer and Beck do report some differences in ratings depending on target pair position. However, since, among other things, the model lacks a retina that might include better central acuity, the positions of the target are irrelevant for the simulations. Figure 24 shows the parameter maps for representative stimuli for the within-group, neutral, and between-group conditions. The target shape is square for the left maps, and it is circle for the right maps. In total we created parameter maps for 18 images (9 spacing conditions x 2 target pair shape).

The strategic connection pattern (that connects target elements and nothing else) is produced for parameter values colored green. We hypothesize that the area of green parameter values reflect (in part) the ease with which the appropriate connections can be created by an observer for a given image. For each spacing condition, if a particular pair of parameters resulted in the strategic connection pattern for both the square and circle targets, then this parameter pair counted as one unit area. The sum of this area corresponded to the model measure of grouping strength. 
This procedure was completed for each spacing condition to produce the plot on the right side of Figure 23b, which shows the model output for each condition. Although there are differences between the pattern of model area and the human ratings, both show the key properties: highest ratings for the within-group conditions and lowest ratings for the between-group conditions. We should note that for the between-group conditions, the model was unable to find any parameter values that produced the desired connection pattern (so the area is zero). Since the data suggest some variability in ratings even for those conditions, there must be additional factors that influence subjective ratings. Nevertheless, just considering the ease of creating the strategic connection pattern already accounts for much of the data.

\section{Conclusions}

The modified bipole circuit provides a model of how an observer can use top-down control to group elements in a multitude of ways via connections. As shown in Figure 19, the connection parameters of this circuit can be tuned so as to connect stimulus elements and, thereby, group stimuli by connections in ways that reflect grouping by proximity, similarity of orientation, similarity of size, similarity of shape, closure, and symmetry. Additionally, the texture and illusory contour demonstration tasks suggest that observers tune these parameters to implement a connection strategy that promotes performance on a given task. Finally, the subjective ratings simulation indicates that the set size of parameter values that produce a particular connection pattern, i.e., one which promotes performance on this task, generally reflects reported subjective ratings of target pair grouping strength.

How do these connections relate to Gestalt grouping principles? Strictly speaking, the model neither obeys Gestalt grouping principles nor directly implements them by, e.g., recognizing that pairs of adjacent objects are of the same size or shape. The model does not know what shape or size are. Instead, it can only detect and manipulate edges. To produce connections between edges, the connection circuits use information about their arrangement, i.e., whether edges are (1) appropriately aligned, (2) an appropriate distance apart, (3) of a particular orientation, and (4) of a particular length. To see how the model uses these properties to produce groupings, consider some examples in Figure 19. In the grouping by proximity examples, some positive spread controller durations allow connections to spread between edges that are aligned and that are within a partic- 

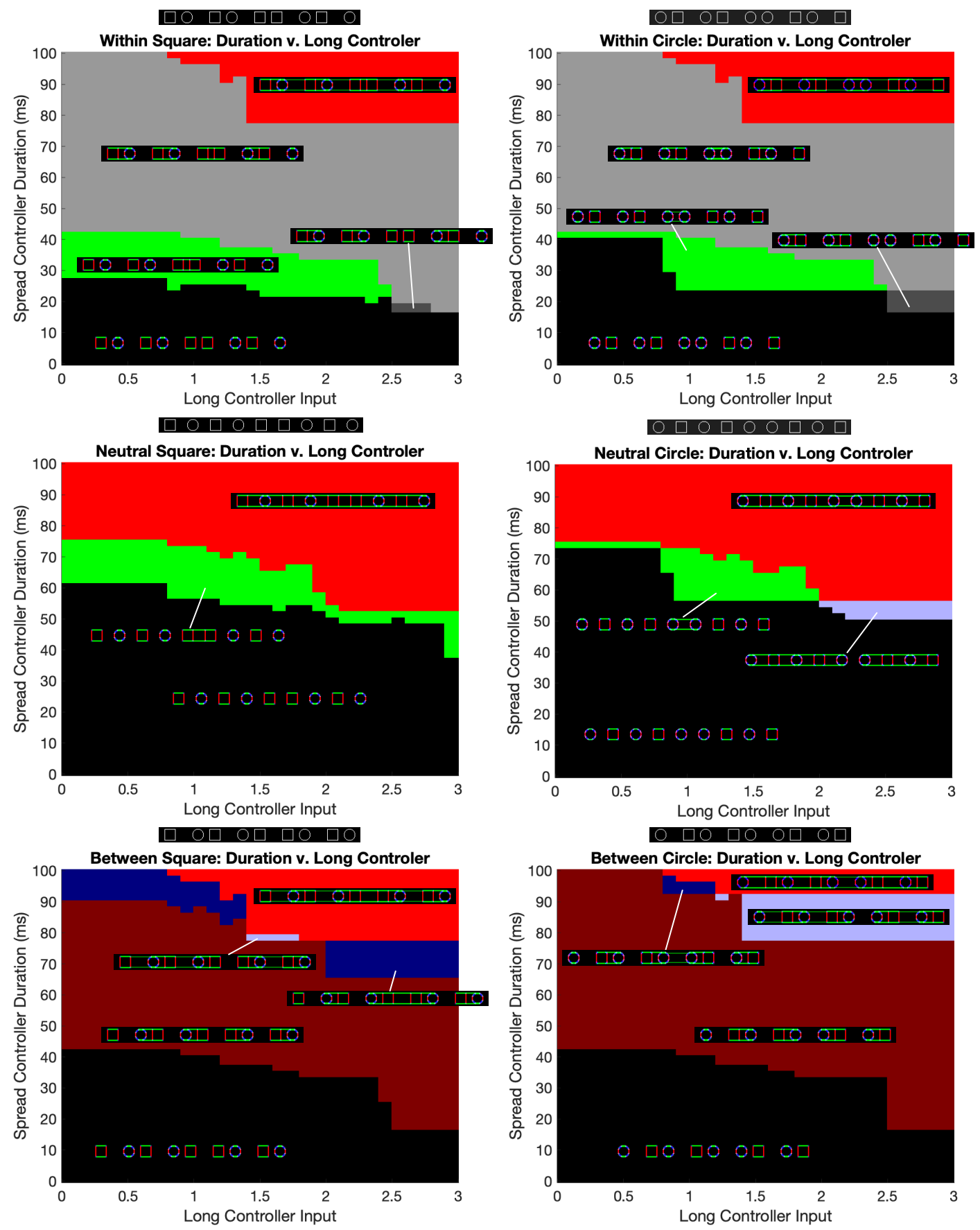

Figure 24: Parameter maps for connection patterns for some of the stimuli used by Palmer and Beck (2007) shown above each map. Duration was in increments of $1 \mathrm{~ms}$, and long controller input was in increments of 0.1. The green regions indicate parameter combinations that produce strategic connections, i.e., connections only between the target pair. The intersection of the green areas in row 1 corresponds to the top right data point in Figure 24 b, that of row 2 corresponds to the central data point, and that of row 3 to the bottom left data point. 
ular distance. In the grouping by orientation examples, some positive spread controller durations allow connections to spread between edges that are aligned and are of the same orientation, while the long/short controller may also be in play to prevent aligned but relatively long edges, e.g., the horizontal bar in the row 3 example in Figure 19, or short edges, e.g., the top of the vertical line and the top of the diagonal line in the row 4 example, from connecting to other edges. In the grouping by similarity of size examples, some positive spread controller durations allow connections to spread between edges that are aligned, while the short controller prevents the shorter edges of the small shapes from connecting to the partially aligned edges of large shapes. In the grouping by shape examples, spread controller duration is again used to promote connections between aligned edges, while the short and long controllers may be used to prevent the edges of particular shapes from connecting, e.g., the short controller prevents the short horizontal edges of the $\mathrm{H}$ and $\mathrm{X}$ from connecting, while the long controller prevents the long top edge of a square from connecting with the top edge of a circle. Thus, fundamentally, the model uses information about properties (1)-(4) to connect particular elements.

Although not an instantiation of Gestalt principles, some of these properties are relatable to properties identified by the Gestalt grouping principles. Property (2) reflects the distances between the elements and, thus, proximity. Property (4) is, arguably, a function of shape for the similarity of shape examples set, e.g., the top edge of a square (circle) is the same length as that of the other square (circle) in the stimulus yet is much longer (shorter) than the top edge of a circle (square). And, size could be regarded as a function of (1) alignment of edges and (4) relative length, e.g., the pair of small circles have shorter edges and the tops and bottom edges of this pair are well aligned while the large circles have larger edges that are well aligned. However, it seems that properties (1), (2), and (3) are involved in all of these examples: creating and manipulating these connections require that the edges are appropriately aligned, be within a certain distance apart (which can be altered by top-down control depending on the spread controller duration and onset), and be of a particular orientation (which can be altered by top-down control depending on which orientations have a positive spread controller duration).

In sum, at least in view of the examples given above, the model indicates that there are several properties of the edges of stimulus elements that allow an observer to group them by connections. The elements that reflect expected groupings given classic grouping principles connect due to the combination of these edges having particular properties and a set of connection parameters that 
are tuned so as to connect particular edges. Although some of these properties relate to Gestalt grouping principles, there is not a clear one-to-one mapping between each property and a Gestalt principle. Thus, it seems that Gestalt grouping principles are high level descriptions of output (i.e., particular patterns of connected elements) that result from simpler underlying mechanisms, which only use information about the arrangement of edges, implemented in the model.

By tuning four top-down control connection parameters, the modified bipole circuit provides a way to implement various connection strategies (i.e., which stimulus elements to connect) including those described by various Gestalt grouping principles as well as those involved in some demonstration tasks involving textures and illusory contours. Additionally, we have shown how the model can be used to account for much of the subjective ratings of grouping strength reported by Palmer and Beck (2007). In turn, the model has the potential to account for performance on a variety of other tasks designed to investigate perceptual grouping and may be used to improve machine vision algorithms (Stettler \& Francis, 2018).

\section{Disclosures}

All authors contributed in a significant way to the manuscript and have read and approved the final manuscript. All authors report that there are no conflicts of interest for this work. Some of these results were presented at the 2019 and 2020 annual meetings of the Vision Sciences Society.

Source code for all simulations can be found at the Open Science Framework https://osf.io/3kmzc/?view_only=6b75ee7702e14bc3a85e23f48fa0a2da.

\section{Acknowledgements}

This work used the Extreme Science and Engineering Discovery Environment (XSEDE), which is supported by National Science Foundation grant number ACI-1548562, Jetstream at IU/TACC through allocation TG-SOC210003. GF was supported by the European Union's Horizon 2020 Framework Programme for Research and Innovation under the Specific Grant Agreement No. 945539 (Human Brain Project SGA3). 


\section{References}

Beck, J. (1966). Effect of orientation and of shape similarity on perceptual grouping. Perception Es Psychophysics, 1, 300-302.

Beck, J., Prazdny, K. \& Rosenfeld, A. (1983). A theory of textural segmentation. In J. Beck, B. Hope, and A. Rosenfeld (Eds.), Human and Machine Vision (pp. 1-38). New York: Academic Press.

Field, D. J., Hayes, A., \& Hess, R. F. (1993). Contour integration by the human visual system: Evidence for a local "association field". Vision Research, 33(2), 173-193.

Francis, G. (2009). Cortical dynamics of figure-ground segmentation: Shine-through. Vision Research, 49, 140-163.

Francis, G., Grossberg, S., \& Mingolla, E. (1994). Cortical dynamics of feature binding and reset: Control of visual persistence. Vision Research, 34, 1089-1104.

Francis, G., Manassi, M., \& Herzog, M. H. (2017). Neural dynamics of grouping and segmentation explain properties of visual crowding. Psychological Review, 124(4), 483-504.

Francis, G., \& Wede, J. (2010). Properties of long-range illusory contours produced by offset-arcs. Perception, 30, 1466-1475.

Gewaltig, M.-O., \& Diesmann, M. (2007). NEST (Neural Simulation Tool). Scholarpedia 2(4), 1430.

Grossberg, S. (1994). 3-D vision and figure-ground separation by visual cortex. Perception $\mathscr{E}$ Psychophysics, 55, 48-120.

Grossberg, S. (2021). Conscious mind, resonant brain: How each brain makes a mind. New York: Oxford University Press.

Grossberg, S., \& Mingolla, E. (1985a). Neural dynamics of perceptual grouping: Textures, boundaries, and emergent segmentations. Perception \& Psychophysics, 38, 141-171.

Grossberg, S., \& Mingolla, E. (1985b). Neural dynamics of form perception: Boundary completion, illusory figures, and neon color spreading. Psychological Review, 92, 173-211. 
Grossberg, S., \& Raizada, R. D. S. (2000). Contrast-sensitive perceptual grouping and object-based attention in the laminar circuits of primary visual cortex. Vision Research, 40, 1413-1432.

Kanizsa, G. (1974). Contours without gradients or cognitive contours? Italian Journal of Psychology, 1, 93-113.

Kirchberger, L., Mukherjee, S., Schnabel, U. H., van Beest, E. H., Barsegyan, A., Levelt, C. N., Heimel, J. A., Lorteije, J. A. M., van der Togt, C., Self, M. W., \& Roelfsema, P. R. (2021). The essential role of recurrent processing for figure-ground perception in mice. Science Advances, $\mathbf{7}$, eabe1833.

Köhler, W. (1925). An aspect of Gestalt psychology. The Pedagogical Seminary and Journal of Genetic Psychology, 32(4), 691-723.

Locher, P. J., \& Wagemans, J. (1993). Effects of element type and spatial grouping on symmetry detection. Perception, 22, 565-587.

Machilsen, B., Pauwels, M., \& Wagemans, J. (2009). The role of vertical mirror symmetry in visual shape detection. Journal of Vision, 9(12), 1-11.

Palmer, S. E. (1999). Vision Science: Photons to Phenomenology. Cambridge, MA: MIT Press.

Palmer, S. E. (2002). Perceptual organization in vision. In H. Pashler (Ed.), Stevens' handbook of experimental psychology: Vol. 1. Sensation and perception (3rd ed., pp. 177-234). New York, NY: Wiley.

Palmer, S., \& Beck, D. (2007). The repetition discrimination task: An objective method for studying perceptual grouping. Perception $\&$ Psychophysics, 69(1), 68-78.

Palmer, S., \& Rock, I. (1994). Rethinking perceptual organization: The role of uniform connectedness. Psychonomic Bulletin $\&$ Review, 1(1), 29-55.

Peterhans, E., von der Heydt, \& R. Baumgartner, G. (1986) Neuronal responses to illusory contour stimuli reveal stages of visual cortical processing. In J. D. Pettigrew, K. J. Sanderson, and W. R. Levick (Eds.), Visual Neuroscience (pp. 343-351). Cambridge, UK: Cambridge University Press. 
Peyser, A., Sinha, A., Vennemo, S. B., Ippen, T., Jordan, J., Graber, S., Morrison, A., Trensch, G., Fardet, T., Mørk, H., Hahne, J., Schuecker, J., Schmidt, M., Kunkel, S., Dahmen, D., Eppler, J. M., Diaz, S., Terhorst, D., Deepu, R.,.. Plesser, H. E. (2017, October 20). NEST 2.14.0. Zenodo. http://doi.org/10.5281/zenodo.882971

Pomerantz, J. R., \& Kubovy, M. (1986). Theoretical approaches to perceptual organization. In R. Boff, L. Kaufman, and J. P. Thomas (Eds.), Handbook of Perception and Human Performance, Vol. 2, Cognitive Processes and Performance (pp. 32-63). New York, NY: Wiley.

Pomerantz, J. R., \& Portillo, M. C. (2011). Grouping and emergent features in vision: Toward a theory of basic Gestalts. Journal of Experimental Psychology: Human Perception and Performance, 37, 1331-1349.

Raizada, R., \& Grossberg, S. (2001). Context-sensitive bindings by the laminar circuits of V1 and V2: A unified model of perceptual grouping, attention, and orientation contrast. Visual Cognition, 8, 431-466.

Rock, I., \& Palmer, S. (1990). The legacy of Gestalt psychology. Scientific America, 263(6), 48-61.

Stettler, M. \& Francis, G. (2018). Using a model of human visual perception to improve deep learning. Neural Networks, 104, 40-49.

Stewart, C. A., Cockerill, T. M., Foster, I., Hancock, D., Merchant, N., Skidmore, E., Stanzione, D., Taylor, J., Tuecke, S., Turner, G., Vaughn, M., \& Gaffney, N. I. (2015). Jetstream: a self-provisioned, scalable science and engineering cloud environment. Proceedings of the 2015 XSEDE Conference: Scientific Advancements Enabled by Enhanced Cyberinfrastructure, 1-8. http://dx.doi.org/10.1145/2792745.2792774

Towns, J., Cockerill, T., Dahan, M., Foster, I., Gaither, K., Grimshaw, A., Hazlewood, S. L., Lifka, D., Peterson, G. D., Roskies, R., Scott, J. R., \& Wilkins-Diehr, N. (2014). XSEDE: Accelerating Scientific Discovery. Computing in Science EJ Engineering, 16(5), 62-74. doi:10.1109/MCSE.2014.80

Wagemans, J., Elder, J. H., Kubovy, M. Palmer, S. E., Peterson, M. A., Singh, M., \& von der Hydt, R. (2012). A century of Gestalt psychology in visual perception: I. Perceptual grouping and figure-ground organization. Psychological Bulletin, 138(6), 1172-1217. 
Wertheimer, M. (1923/1950). Laws of organization in perceptual forms. In W. D. Ellis (Ed.), $A$ sourcebook of Gestalt psychology (pp. 71-81). New York: Humanities Press.

Zhang, S., Xu, M., Kamigaki, T., Do, J. P. H., Chang, W.-C., Jenvay, S., Miyamichi, K., Luo, L. \& Dan, Y. (2014). Long-range and local circuits for top-down modulation of visual cortex processing, Science, 345(6197), 660-665. 